\title{
Orientation Selectivity, Preference, and Continuity in Monkey Striate Cortex
}

\author{
Gary G. Blasdel \\ Department of Neurobiology, Harvard Medical School, Boston, Massachusetts 02115
}

\begin{abstract}
Maps of orientation preference and selectivity, inferred from differential images of orientation (Blasdel, 1992), reveal linear organizations in patches, $0.5-1.0 \mathrm{~mm}$ across, where orientation selectivities are high, and where preferred orientations rotate linearly along one axis while remaining constant along the other. Most of these linear zones lie between the centers of adjacent ocular dominance columns, with their short iso-orientation slabs oriented perpendicular, in regions enjoying the greatest binocular overlap. These two-dimensional linear zones are segregated by one- and zero-dimensional discontinuities that are particularly abundant in the centers of ocular dominance columns, and that are also correlated with cytochrome oxidase-rich zones within them. Discontinuities smaller than $90^{\circ}$ extend in one dimension, as fractures, while discontinuities greater than $90^{\circ}$ are confined to points, in the form of singularities, that are generated when orientation preferences rotate continuously through $\pm 180^{\circ}$ along circular paths. The continuous rotations through $180^{\circ}$ imply that direction preferences are not organized laterally in striate cortex. And they also ensure that preferences for all orientations converge at each singularity, with perpendicular orientations represented uniquely close together on opposite sides.
\end{abstract}

The periodic interspersing of linear zones and singularities suggests that orientation preferences are organized by at least two competing schemes. They are optimized for linearity, along with selectivity and binocularity, in the linear zones, and they are optimized for density near singularities. Since upper-layer neurons are likely to have similarly sized dendritic fields in all regions (Lund and Yoshioka, 1991), those in the linear zones should receive precise information about narrowly constrained orientations, while those near singularities should receive coarse information about all orientations-very different inputs that suggest different perceptual functions.

Cells in striate cortex are characterized by two response properties-ocular dominance and orientation selectivity (Hubel and

\footnotetext{
Received Apr. 1, 1991; revised Feb. 16, 1992; accepted Mar. 17, 1992.

This work was supported by National Eye Institute Grant EY05403, the Office of Naval Research, the Klingenstein Foundation, and the McKnight Foundation. I thank David Hubel, Jenny Lund, Terry Sejnowski, Marge Livingstone, and Klaus Obermayer for helpful comments on the manuscript, and Susan Cowdery for expert and cheerful assistance through all phases of this work. Dr. Aniruddha Das participated in some analyses of singularities. Gina Quinn helped with the manuscript. Scan Sommers and Mike Lafratta machined much of the specialized equipment, and Jenny Robbins provided useful guidance during the processing of histological tissue.

Correspondence should be addressed to Gary G. Blasdel, Department of Neurobiology, Harvard Medical School, 220 Longwood Avenue, Boston, MA 02115. Copyright $\odot 1992$ Society for Neuroscience $0270-6474 / 92 / 123139-23 \$ 05.00 / 0$
}

Wiesel, 1962, 1968, 1972, 1974a,b)-that receive much attention on account of their presumed relevance to vision. Ocular dominance is associated with binocularity, the convergence of inputs from both eyes onto single cells that is thought to underlie stereopsis. Even though most cells respond to either eye, they usually are dominated by one. And, in old-world primates, this dominance is thought to reflect the segregated bands of right and left eye inputs in layer $4 \mathrm{c}$ (Hubel and Wiesel, 1972; Blasdel and Lund, 1982). The cells in layer $4 \mathrm{c}$ respond exclusively to the eye providing input, and since the cells above and below arc dominated by this eye as well, slab-shaped ocular dominance domains may be defined, as illustrated in Figure $1 c$ (Hubel and Wiesel, 1972).

Orientation selectivity is associated with edges, and governed by at least two variables - preference and selectivity - which vary. While orientation preferences are aligned vertically (Hubel and Wiesel, 1974a), like ocular dominance, they are organized laterally in ways that have proven difficult to understand. By directing electrodes laterally, through the upper layers, Hubel and Wiesel (1974a) discovered that the preferred orientation rotates linearly with distance (see Fig. $1 a, b$ ). Since it rotates at different rates for different penetrations, they reasoned that it must entail constant values along one horizontal axis and constantly rotating ones along the other. This led them to suggest that orientation preferences might be represented in parallel slabs as well, like those for ocular dominance only narrower (see Fig. 1c). They also suggestcd that orientation and ocular dominance slabs might intersect at right angles.

Hubel and Wiesel's suggestion of extended orientation slabs seemed to be confirmed by 2-deoxyglucose (2DG) (Sokoloff et al., 1977) experiments that revealed the expected slab-shaped patterns of activity in response to single orientations (Hubel et al., 1978; Schoppmann and Stryker, 1981). But their suggestion that ocular dominance and orientation slabs intersect at right angles appeared to be ruled out by the same experiments. Both conclusions had to be reevaluated, however, when it became evident (Horton and Hubel, 1980; Livingstone and Hubel, 1984a) that parts of the 2DG-labeled "orientation columns"-those containing high concentrations of the mitochondrial enzyme cytochrome oxidase (Wong-Riley, 1979)-are not selective for orientation, and that they also have high metabolic rates that make them label indiscriminately, even at rest (Humphrey and Hendrickson, 1983).

While it now is acknowledged that 2DG-labeled orientation bands reflect more than preferences for one orientation, it frequently is assumed that blobs are simply labeled in addition to the "orientation columns" illustrated in Figure $1 c$. Horton and Hubel's (1980) findings imply much more, however. Since cytochrome oxidase blobs are always included in bands of 2DG 


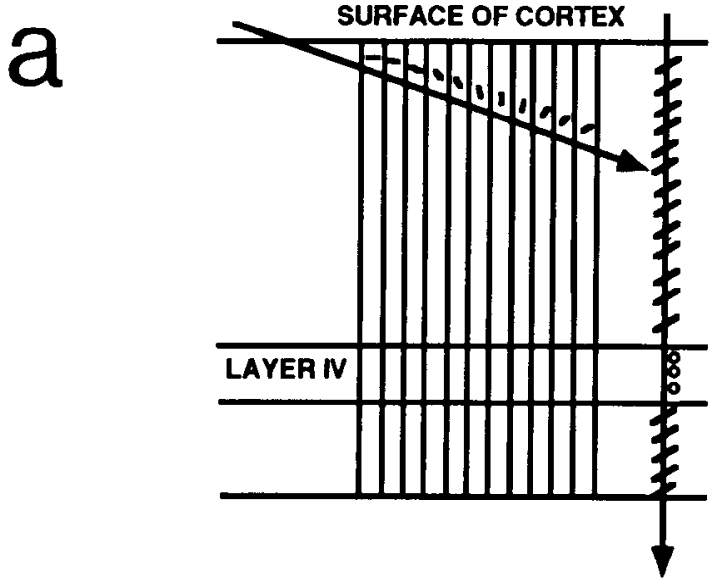

b

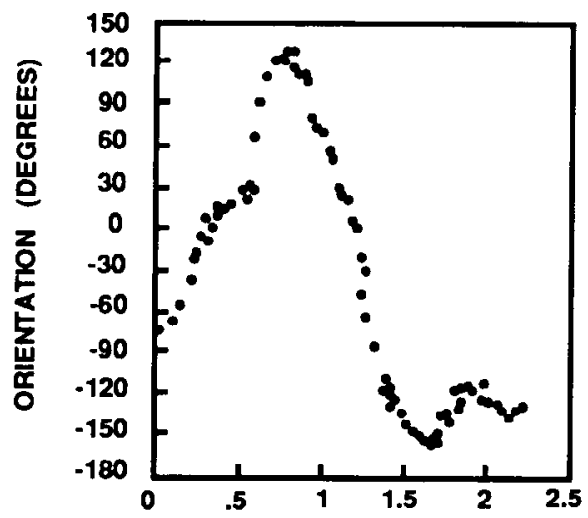

TRACK DISTANCE (MILLIMETERS)
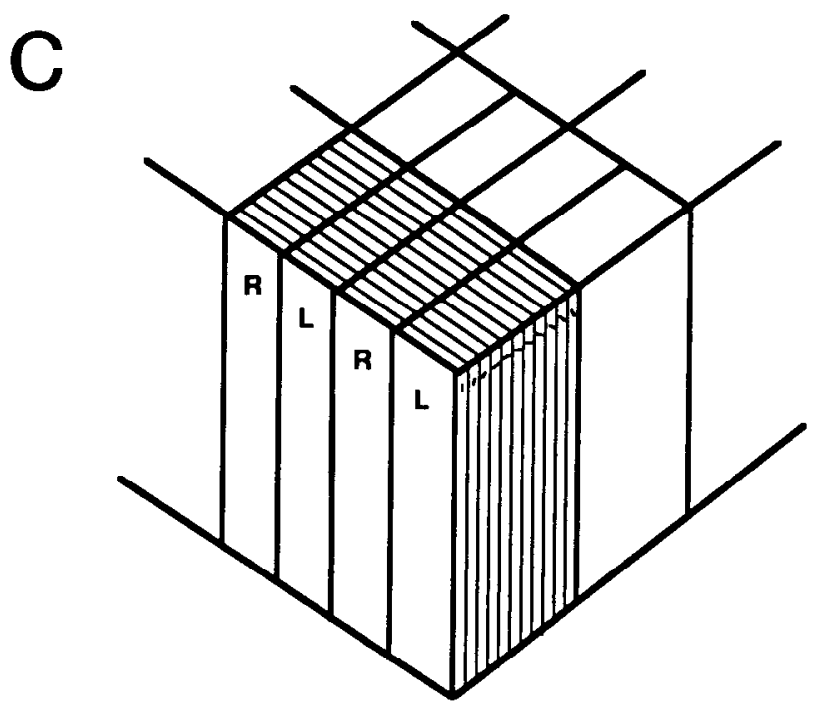

Figure 1. Organizational schcmes for orientation preferences, deduced from microelectrode penetrations (Hubel and Wiesel, 1974a). a, Hubel and Wiesel's results for electrode penetrations conducted perpendicular and parallel to the cortical surface. For perpendicular penetrations, the preferred orientation remains constant (except in layers $4 \mathrm{a}$ and $4 \mathrm{c}$, where the selectivity for orientation is weak or absent). For lateral penetrations, the preferred orientation rotates continuously for distances of $0.5-1.0$ $\mathrm{mm}$. $b$, Orientation preferences plotted as a function of distance for one tangential penetration. Note that orientation preferences rotate linearly label, orientation-selective regions cannot extend farther than spaces between blobs, which are $350 \mu \mathrm{m}$ apart on average. Recent findings (Blasdel, 1992) restrict possible inferences further by revealing that, even between blobs, the centers of 2DGlabeled "orientation" slabs do not always reflect preferences for the orientation used to drive uptake.

The first opportunity for determining orientation preferences accurately, in large areas of cortex, was provided by an optical imaging strategy (Blasdel and Salama, 1986) that can be applied repeatedly in vivo. The preceding companion article (Blasdel, 1992) dealt with the patterns acquired most directly-differential images of ocular dominance and differential images of orientation-as well as with reliability, stability, and artifacts. It also revealed an interesting correlation between orientation selectivity and binocularity. It made no attempt to infer orientation preferences directly, however.

This article describes an algorithm for combining differential images, acquired with different orientations, and uses it to calculate maps of orientation preference and selectivity that are then compared and analyzed. The results support previous observations (Blasdel and Salama, 1986; Blasdel, 1989a), that orientation preferences change linearly in patches, $0.5-1.0 \mathrm{~mm}$ across, and that these patches are separated semiperiodically by discontinuities that are particularly abundant in the centers of ocular dominance columns. A distinction is introduced between discontinuities that are smaller than $90^{\circ}$, which are defined here as fractures, and those that are larger than $90^{\circ}$, which are defined here as singularities, on account of their different distributions. A simple hypothesis, relating the linearity and density of orientation preferences to visual scene segmentation, is then advanced along with suggestions for testing it.

\section{Materials and Methods}

Patterns comparable to those reported here (and in the preceding article) have been obtained from 10 separate animals (one Macaca mulatta, nine Macaca nemestrina), some studies of which have been reported previously (Blasdel and Salama, 1986; Blasdel and Tootell, 1987; Kiorpes and Blasdel, 1987; Blasdel and Haglund, 1989; Blasdel, 1989a,b). While these observations apply to all animals, most are illustrated with images from one, a female Macaca nemestrina, for simplicity and continuity of thought. Differential images of orientation and ocular dominance were explored in the preceding article (Blasdel, 1992), along with technical details about animal preparation and differential video imaging. Some aspects of the latter deserve reemphasis.

Differential imaging. Differential images of orientation are obtained by averaging two images-one positive, one negative-of cortex responding alternately to complimentary stimuli. The negative image is then subtracted from the positive one to reveal places where the optical

over distances of $0.5-1.0 \mathrm{~mm}$, but over intervals longer than this the linearity is broken by reversals in the direction of rotation as well as by abrupt shifts in preference. $c$, "Ice-cube" model of striate cortex, proposed by Hubel and Wiesel (1977) on the basis of results similar to those shown in $b$. This model has four principle components: (1) regions preferring one eye or one orientation project vertically between pia and white matter and accordingly take on the appearance of slabs, (2) slabs representing the two eyes lie parallel to one another, (3) slabs representing different orientations lie parallel to one another, and (4) the two set of slabs (representing the two eyes and different orientations) intersect at a consistent angle. Doubt was cast on this last component by experiments with the metabolic marker 2DG, which showed 2DG-labeled "iso-orientation" bands intersecting ocular dominance bands at many different angles (Hubel et al., 1978). But these experiments have themselves been called into question by more recent observations that the "orientation bands" labeled with 2DG include regions nonselective for orientation (Horton and Hubel, 1980; Livingstone and Hubel, 1984). These illustrations are taken from Hubel and Wiesel (1974a, 1977). 


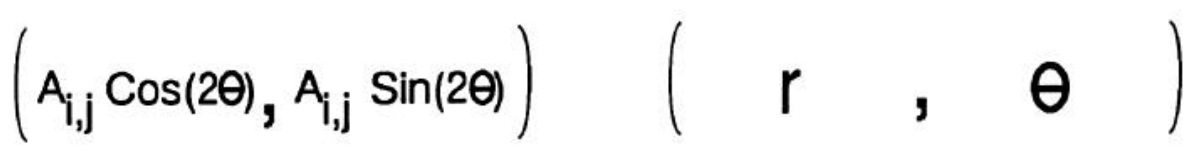
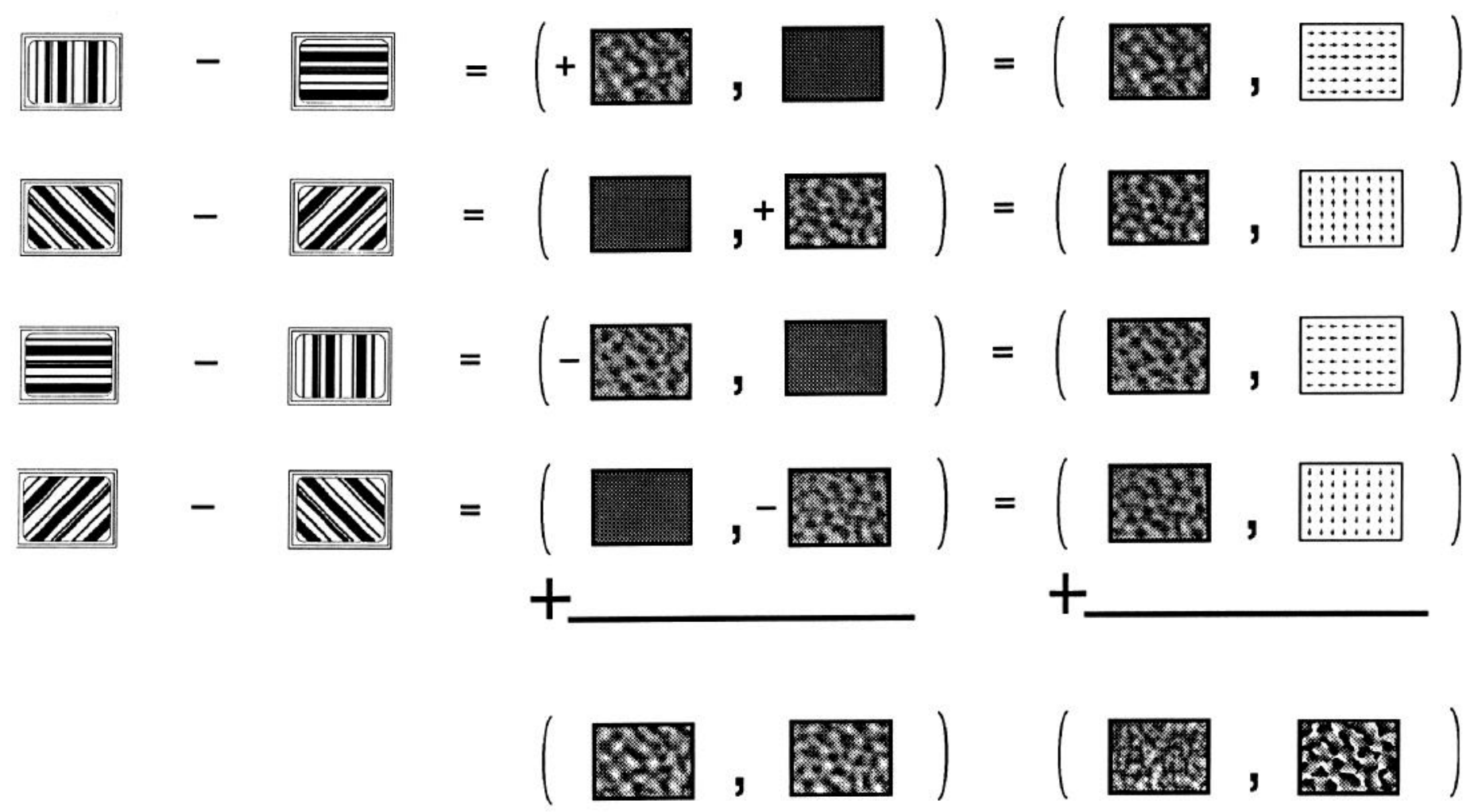

Figure 2. This figure illustrates the algorithm used to extract and add orientation-dependent components from each differential image of orientation. Because the scalar values at each image location indicate preferences for one orientation or the other (orthogonal orientation), with positive or negative values, they can be converted into vectors by multiplying each one with a unit vector angled at twice the stimulus orientation. The orientation must be doubled because vectors (which are directed) and orientations (which are not) cycle back on themselves after rotating through $360^{\circ}$ and $180^{\circ}$, respectively, and therefore can only be equated when orientation angles are doubled. This has the added benefit that most different orientations (those that are orthogonal) are represented by most different vectors (those rotated through $180^{\circ}$ ), which cancel. Different rows indicate responses to different pairs of orthogonal contours, and how the resulting differential images are treated. The middle and right drawings illustrate conversions to vectors, displayed as cosine and sine pairs, in Cartesian coordinates, and as magnitudes and angles in Polar coordinates. Once differential image values have been converted in this way, they can be added at corresponding positions, to yield output vectors that correspond (in size and direction) to orientation selectivity and preference (times two) at each location.

absorption changed in response to visual stimulation. In the case of orientation, the complimentary stimuli consist of orthogonally oriented contours moving bidirectionally at $1.5 \% \mathrm{sec}$. Positive (dark) values indicate preferences for the test orientation, while negative (light) values indicate preferences for the orthogonal orientation. Neutral values indicate no preference for either orientation.

Strong signals make strong assertions, whether positive or negative, because they only can derive from regions that are responsive as well as selective. Weak or neutral signals are ambiguous for reciprocal reasons, since they could derive from weak responses or a lack of selectivity. The apparent confusion can be resolved in many cases with other differential images. Regions expressing no preference for vertical or horizontal, for example, might express a strong preference for right or left oblique, or regions responding to all orientations (e.g., near cytochrome oxidase blobs) might be strongly dominated by one eye. In either case, one can feel reasonably confident that the neutral zones reflect a lack of selectivity rather than a lack of responsiveness.

Vectoral conversion. When differential images are acquired with different pairs of orthogonal contours $-0^{\circ} / 90^{\circ}$ and $15^{\circ} / 105^{\circ}$, for examplethey cannot be added directly because they reflect preferences for different orientations. Information common to both can be extracted, however, if the differences in orientation are taken into account. This is done most easily by transforming values at every location, in every image, into vectors (Fig. 2), with angles corresponding to twice the positive stimulus orientation, and lengths corresponding to net intensities. Intensity values in images derived from vertical/horizontal $\left(0^{\circ}\right.$ $\left.90^{\circ}\right)$, left/right oblique $\left(45^{\circ} / 135^{\circ}\right)$, and horizontal/vertical $\left(90^{\circ} / 0^{\circ}\right)$, for example, can be multiplied by unit vectors with angles of $0^{\circ}, 90^{\circ}$, and $180^{\circ}$, respectively. Since negative values correspond to vectoral rotations through $180^{\circ}$, preferences for orthogonal orientations (which, by definition, are offset by $90^{\circ}$ ) cancel automatically.

Once the differential images have been transformed, the resulting vectors can be added to reflect orientation-weighted contributions from each image. Because stimulus orientations are multiplied by two, contributions generated by similar orientations reinforce one another, while those generated by orthogonal orientations cancel. The angles and magnitudes of summed vectors then reflect orientation preferences (multiplied by two) and selectivities (or responsiveness) in corresponding areas of cortex.

Orientation gradient. Orientation preferences in this article are analyzed with respect to their gradient, which, like a first derivative, measures the rate of change at every point but, unlike a first derivative, is defined in two dimensions. For the results reported here, the gradient was calculated by taking partial derivatives of orientation with respect to $x$ and $y$, and converting the result to Polar coordinates. At pixel location $(i, j)$, for example, $\partial \theta / \partial x$ is estimated from $\Delta \theta / \Delta x \approx\left(\theta_{i+1 j}-\right.$ $\left.\theta_{i, j}\right)$, and $\partial \theta / \partial y$ from $\Delta \theta / \Delta y \approx\left(\theta_{i, j+1}-\theta_{i, j}\right)$. If the absolute magnitude of either $\Delta \theta / \Delta x$ or $\Delta \theta / \Delta y$ exceeds $90^{\circ}, 180^{\circ}$ is added (or subtracted) to bring 

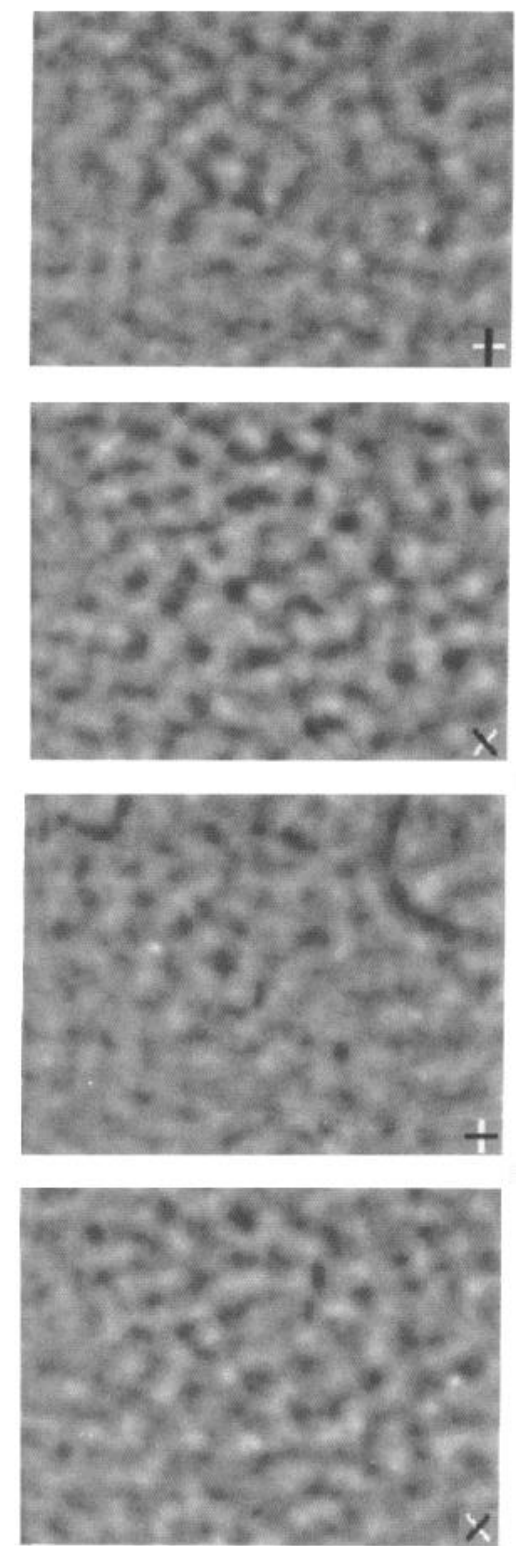
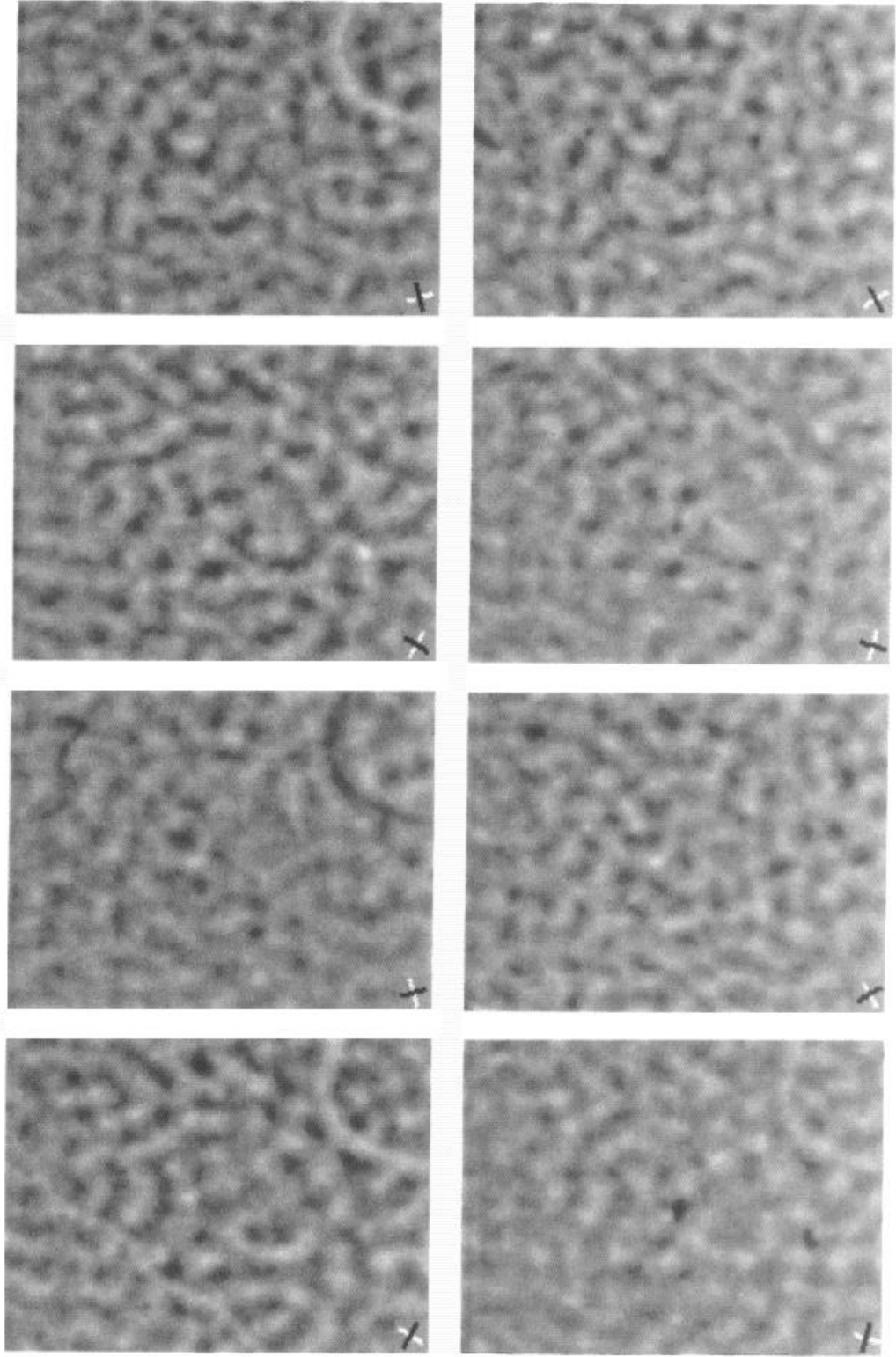

$1 \mathrm{~mm}$

Figure 3. Differential images of orientation achieved at low magnification with 12 different pairs of orthogonal contours. The horizontal axis in each frame corresponds to $8 \mathrm{~mm}$. All images were acquired with orthogonal pairs of oriented contours, moving bilaterally (in opposite directions during even and odd trials) at $1.5 \%$ sec. The dark and light values in each image reflect preferences for each of the 12 pairs of orthogonal contours and are indicated in the lower right corner by crossed dark and light bars. Successive frames were collected with contour pairs rotated in increments of $15^{\circ}$, and reflect response differences for $0^{\circ}-90^{\circ}, 15^{\circ}-105^{\circ}, 30^{\circ}-120^{\circ}, 45^{\circ}-135^{\circ}, 60^{\circ}-150^{\circ}, 75^{\circ}-165^{\circ}, 90^{\circ}-0^{\circ}, 105^{\circ}-15^{\circ}, 120^{\circ}-30^{\circ}, 135^{\circ}-45^{\circ}, 150^{\circ}-60^{\circ}$, and $165^{\circ}-75^{\circ}$.

it within $\pm 90^{\circ}$, because the preferred orientation rotates through a complete cycle in $180^{\circ}$, making $0^{\circ}$ and $180^{\circ}$ equivalent. As a direct consequence, there can be no jumps greater than $90^{\circ}$ along any single axis. Results are then converted into Polar coordinates $(r, \phi)$, by letting $r_{i,}=$ $\left\{(\partial \theta / \partial x)^{2}+(\partial \theta / \partial y)^{2}\right\}^{1 / 2}$, and $\phi_{i j}=\tan ^{-1}\{(\partial \theta / \partial y) /(\partial \theta / \partial x)\}$. For the Results and Discussion, attention is focused on the magnitude $\{r\}$ of this vector, which indicates the steepest rate of change at any point, irrespective of direction.

Since the gradient is a linear operator, it technically is undefined in regions where orientation preferences change discontinuously. The operations used to calculate the gradient from discrete values $(\Delta \theta / \Delta x$ and $\Delta \theta / \Delta y$ ) nevertheless generate large numbers at these locations-numbers (more accurately referred to as $\Delta \theta / \Delta r$, where $r$ is a unit length vector) that can be used to find discontinuities as well as to indicate their size, even though technically they are not part of the gradient. When these values are visualized, discontinuous changes appear as short white lines or dots, running across regions of continuous tone (see Fig. $8 a$ ). Note that, even though $\Delta \theta$ cannot exceed $90^{\circ}$ anywhere along a line of discontinuity, it can exceed $90^{\circ}$ momentarily at singularities, where $\Delta \theta / \Delta x$ and $\Delta \theta / \Delta y$ both approach $90^{\circ}$, and where $\Delta \theta$ may therefore achieve values as high as $\left(90^{2}+90^{2}\right)^{1 / 2} \approx 127^{\circ}$ (per pixel).

Definitions. Since many terms in this article are used commonly to refer to different things (e.g., orientation selectivity, which is used to indicate tuning as well as preferred orientation), their intended meanings are provided below.

Orientation preference: the orientation yielding the strongest response. Since direction selectivity is not a factor (see Results), contours rotated through $180^{\circ}$ are equivalent. 

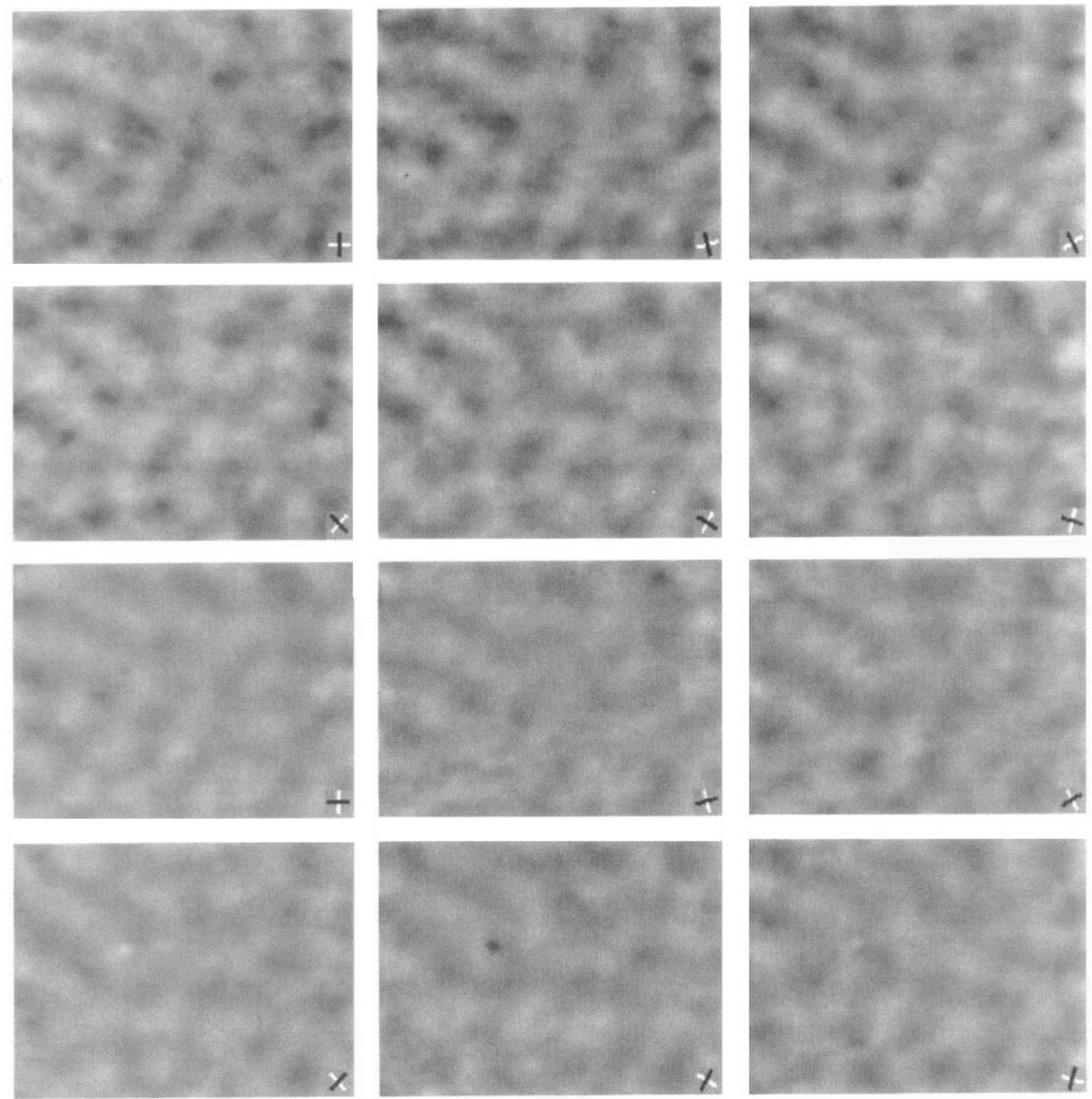

\section{$1 \mathrm{~mm}$}

Figure 4. Differential images of orientation achieved at high magnification with 12 different pairs of orthogonal contours. The horizontal axis in each frame corresponds to $4.4 \mathrm{~mm}$. As in Figure 3, all images were acquired with orthogonal pairs of oriented contours moving bilaterally at $1.5 \%$ sec. Dark and light values in each frame reflect preferences for each of two orthogonal contours, which are indicated by dark and light bars in the lower right corner, and which are rotated by $15^{\circ}$ in successive frames. Therefore, these images reflect response differences for $0^{\circ}-90^{\circ}, 15^{\circ}-105^{\circ}, 30^{\circ}-$ $120^{\circ}, 45^{\circ}-135^{\circ}, 60^{\circ}-150^{\circ}, 75^{\circ}-165^{\circ}, 90^{\circ}-0^{\circ}, 105^{\circ}-15^{\circ}, 120^{\circ}-30^{\circ}, 135^{\circ}-45^{\circ}, 150^{\circ}-60^{\circ}$, and $165^{\circ}-75^{\circ}$.

Orientation selectivity: the rate at which responses fall to zero with increasing displacement from the preferred orientation. Neurons with high selectivity respond over narrow ranges of orientation, while neurons with low selectivity respond over broad ranges. Neurons with no selectivity respond to all orientations.

Break: a zero-dimensional (punctate) discontinuity in the orientation preferences encountered along a line (in one dimension).

Fracture: a one-dimensional rift in orientation continuity across a surface (in two dimensions), where preferences change by $90^{\circ}$ or less.

Singularity: a zero-dimensional discontinuity in two dimensions, arising where orientation preferences rotate continuously through $\pm 180^{\circ}$ in a circle. Even though orientation preference is undefined at the center, there is a discontinuity implied that exceeds $90^{\circ}$.

Orientation density: range of orientations preferred per unit area, which in this article is assumed to be that covered by the dendrites of a "typical" upper-layer cell with a cylindrical field $300 \mu \mathrm{m}$ in diameter. Contour: a single, isolated edge, extending along a well-defined axis in one dimension.

Surface: a two-dimensional distribution of color and/or edges.

\section{Results}

Figures 3-5 show differential images of orientation from three separate animals. Dark and light shadings reflect preferences for the orientations indicated by dark and light bars in the lower right corners. As one can see by comparing successive frames, 

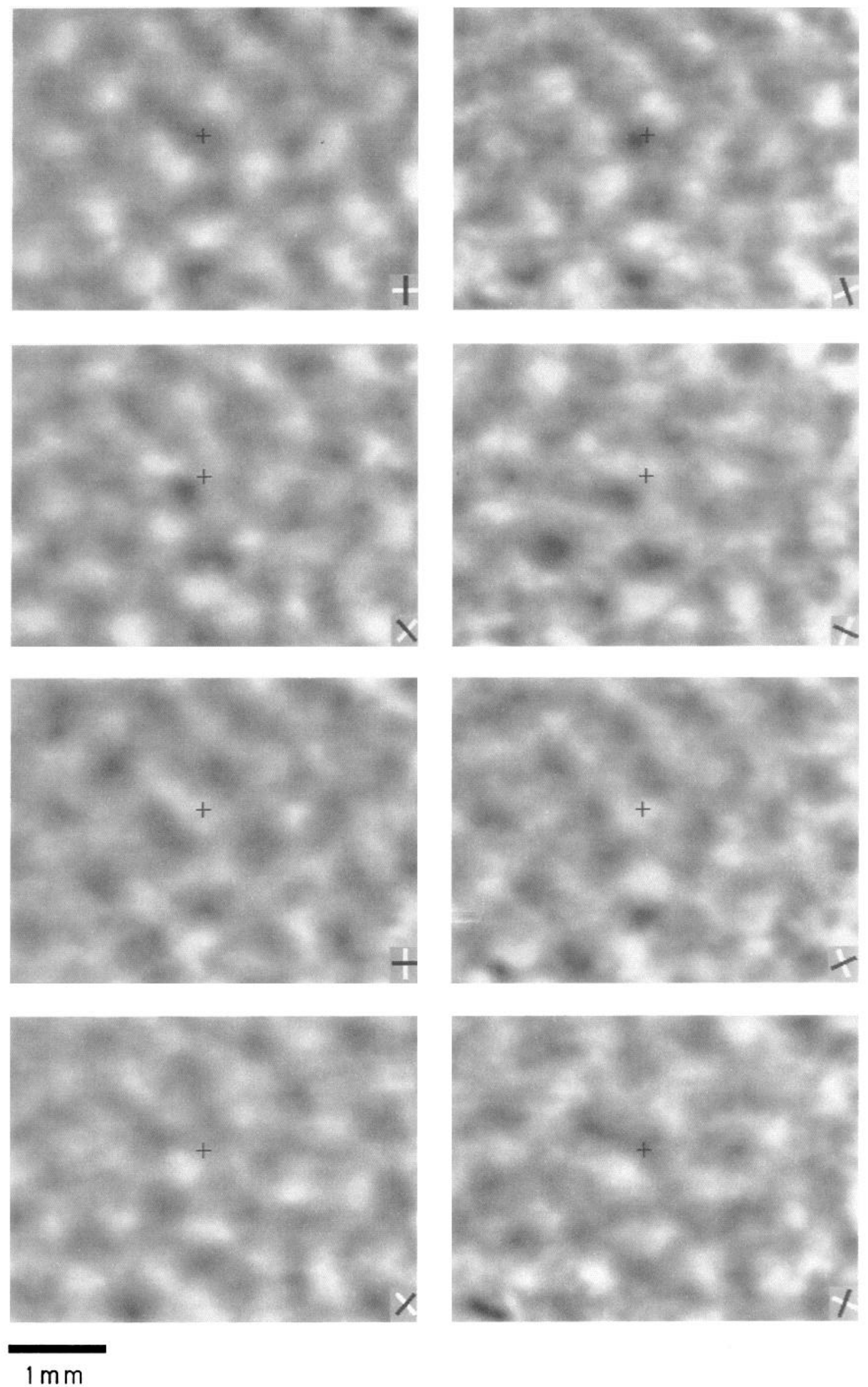

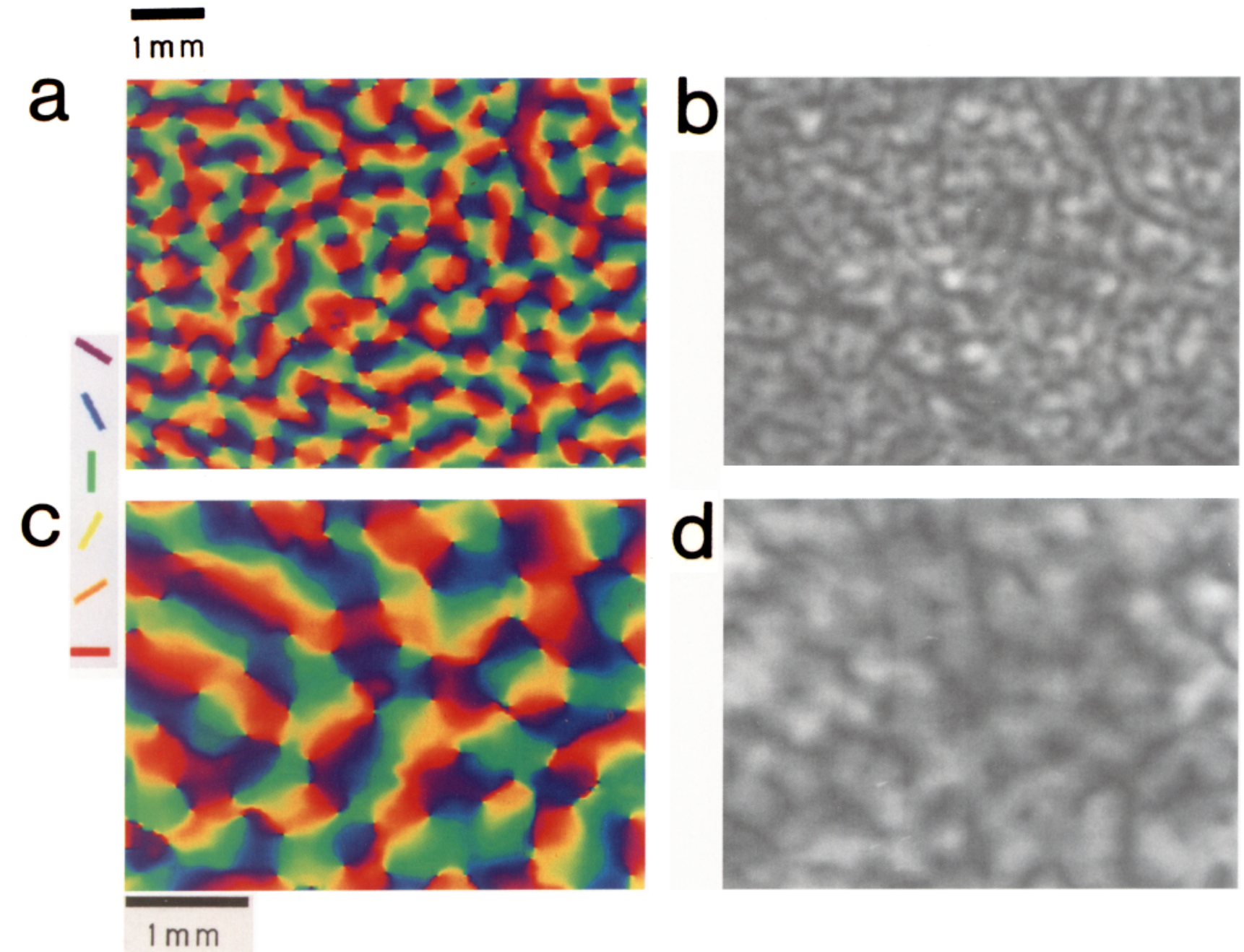

Figure 6. When the differential images in Figures 3 and 4 are combined according to the rules illustrated in Figure 2, they produce a field of output vectors, the two components of which in Polar coordinates (right-hand vectors in Fig. 2) correspond to orientation preference ( $2 \times)$ and selectivity, which appear separately in $a$ and $b$, for the images in Figure 3 , and in $c$ and $d$, for the images in Figure 4. The orientation preferences in $a$ and $c$ must be illustrated in color because they cycle continuously through $180^{\circ}$. Complimentary colors have been chosen to indicate orthogonal orientations: green and red indicate preferences for vertical and horizontal, while blue and yellow indicate preferences for left and right oblique. Values in $b$ and $d$ are indicated in gray, with lighter regions more selective (and responsive) than darker ones. As noted in the Results, light values are unambiguous - they indicate strongly responsive regions that were highly selective-while darker values might indicate regions that were nonresponsive as well as ones that were nonselective. Since many of the dark regions are aligned with ocular dominance centers, though, where responses to one or the other eye are pronounced, they would appear to indicate a lack of orientation selectivity since a lack of responsiveness can be ruled out.

the darkest and lightest regions shift laterally as visual stimuli rotate. While this agrees with previous observations, that orientation preferences change linearly with distance (Hubel and Wiesel, 1974a), the actual organization of preferences is difficult to infer from any particular image because light and dark bands can arise from factors other than slabs of cells preferring the same orientation (Blasdel, 1992).

The information needed to calculate orientation preferences and selectivities is nevertheless present in all images collectively, and can be calculated by estimating vertical/horizontal and left/ right oblique components for each one and summing them separately. The resulting vectors are then converted from Cartesian to Polar coordinates. The orientation preferences and selectivities derived in this manner appear in Figure 6, $a$ and $b$, for the images in Figure 3, in Figure 6, $c$ and $d$, for the images in Figure 4 , and in Figure 7, $a$ and $b$, for the images in Figure 5. As one can see, the patterns in Figures 6, $a$ and $c$, and $7 a$ are all remarkably similar, despite differences in magnification. The accuracy and repeatability of this analysis are discussed further at the end of Results, along with potential artifacts.

Figure 5. Differential images of orientation achieved at high magnification with eight different pairs of orthogonal contours. The horizontal axis in each frame corresponds to $4.4 \mathrm{~mm}$. As in Figures 3 and 4 , all images were acquired with orthogonal pairs of contours moving bilaterally at $1.5 \%$ sec. Dark and light values in each frame reflect preferences for each of two orthogonal contours, which are indicated by dark and light bars in the lower right corner, and which are rotated by $22.5^{\circ}$ in successive frames. Therefore, these images reflect response differences for $0^{\circ}-90^{\circ}, 22.5^{\circ}-112.5^{\circ}$, $45^{\circ}-135^{\circ}, 67.5^{\circ}-157.5^{\circ}, 90^{\circ}-0^{\circ}, 112.5^{\circ}-22.5^{\circ}, 135^{\circ}-45^{\circ}$, and $157.5^{\circ}-67.5^{\circ}$. 

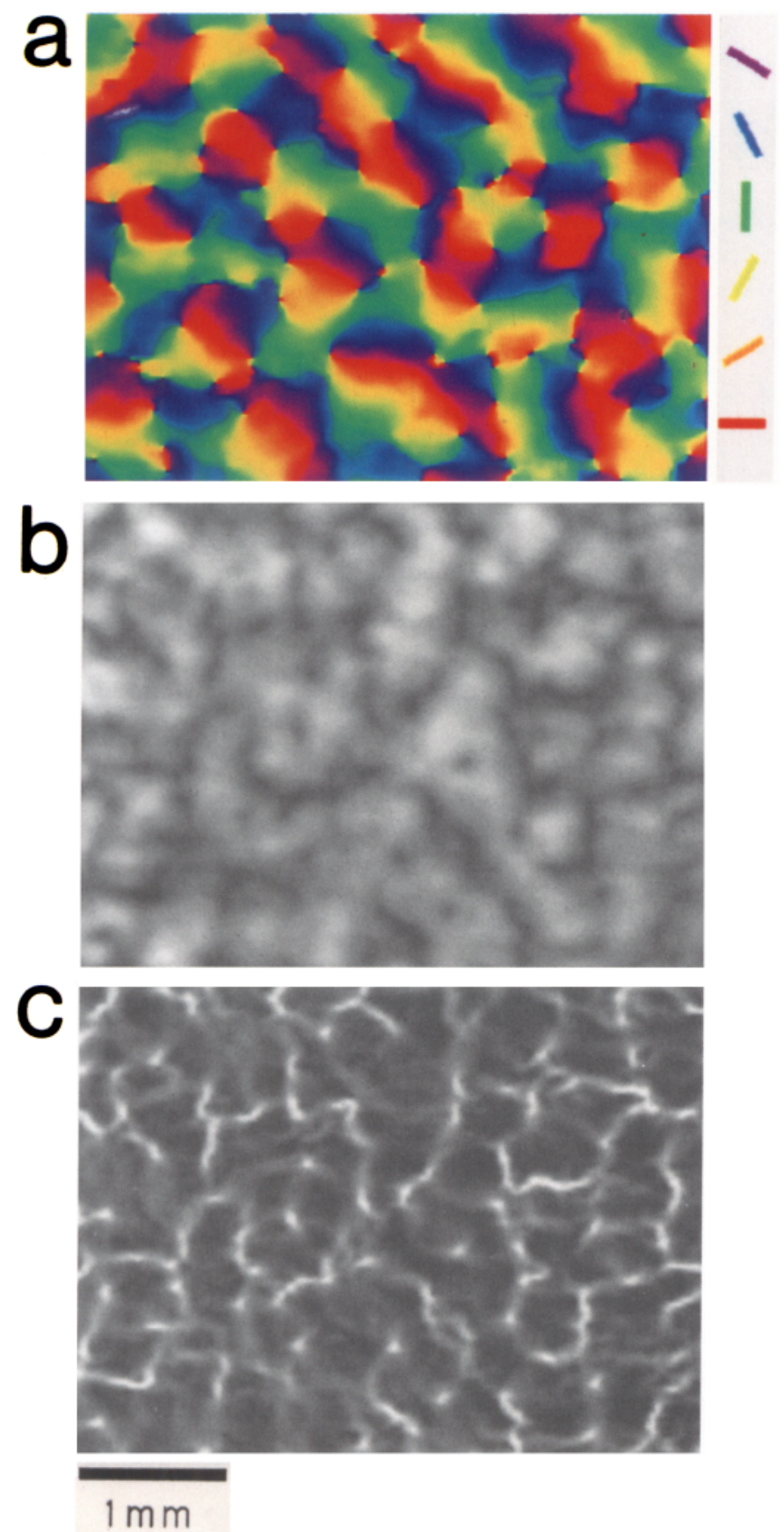

Figure 7. When the differential images in Figure 5 are combined, they produce similar maps of orientation preference and selectivity, which appear in $a$ and $b$. As in Figure 6, orientation preferences $(a)$ must be illustrated in color because they cycle continuously through $180^{\circ}$. Complimentary colors indicate orthogonal orientations: green and red indicate preferences for vertical and horizontal, while blue and yellow indicate preferences for left and right oblique. Orientation selectivity (b) is indicated in gray, with lighter and darker regions indicating more and less selectivity. As noted in the Results, light values are unambiguous - they indicate strongly responsive regions that were highly selective-while darker values might indicate regions that were nonresponsive as well as ones that were nonselective. Since many of the dark regions are aligned with ocular dominance centers, though, where responses to one or the other eye are pronounced, they would appear to result from a lack of orientation selectivity since these regions obviously are responsive to the visual stimuli used. In $c$, one sees the absolute magnitude of the orientation gradient at each location, which corresponds to the rate at which orientation preferences are changing between pixels. While the gradient is a linear operator, defined only in regions of continuous change, it is calculated from discrete values that change

\section{Orientation preference}

We are now in a position to examine the spatial distribution of orientation preferences. Hubel and Wiesel's (1974a) suggestion, that cells preferring slightly rotated orientations are organized in extended orientation slabs, would be supported if the colored regions in Figure $7 a$ were joined in long, rainbowlike patterns. The observed patterns are more complicated, however. Rainbowlike structures are apparent locally, in patches $0.5-1.0 \mathrm{~mm}$ across, but iso-orientation slabs longer than $0.5 \mathrm{~mm}$ are rare, as are linear sequences (of slightly rotated orientation preferences) longer than $1.0 \mathrm{~mm}$.

\section{Linearity, fractures, and singularities}

Further insights are achieved by calculating the rate at which orientation preferences change locally - a rate given by the magnitude of the gradient (see Materials and Methods). As one can see in Figure $7 c$, where higher rates of change are indicated by lighter values, most of the surface is characterized by slow (dark) and continuous rates of change, interrupted semiperiodically by bright lines (fractures) and points (singularities) that fragment the continuous-toned area into patches. It is unclear at the moment whether these patches should be considered separately, as isolated domains, or as part of a larger continuum since one can trace continuous paths around most disruptions. The arrangements are such that linear sequences longer than $0.5-1.0 \mathrm{~mm}$ are difficult to find, however.

The lines of discontinuity were discovered by Blasdel and Salama (1986), who called them "fractures" on account of their broken and incomplete appearance. They are two-dimensional extensions of the "breaks in sequence regularity" described by Hubel and Wiesel (1974a). Even though they are virtually continuous along their trajectories, some fractures are themselves interrupted by brief intensifications, where the gradient exceeds $90^{\circ}$. These points are induced by singularities (see below) where all orientation preferences converge (Blasdel, 1989a).

Particularly large gradient values are indicated over the map of orientation preferences in Figure $8 a$, where the correspondence between fractures, singularities, and the boundaries of linear zones is obvious. Since orientation preferences change linearly in patches $0.5-1.0 \mathrm{~mm}$ across, stacks of parallel isoorientation slabs can be identified easily at this scale, which is the one most likely to have dominated previous investigations (Hubel and Wiesel, 1974a).

\section{Orientation selectivity}

The orientation selectivities indicated by vector magnitudes are indicated in Figure $7 b$, where the lightest regions, reflecting the greatest selectivities, also cluster in two-dimensional patches. These are separated by darker, one-dimensional borders where orientation selectivity is weak or absent. Superficially, therefore, the distribution of selective and nonselective regions resembles

$\leftarrow$

in any case and that can be used to visualize discontinuities. As one can see in this image, discontinuous changes either occur alone, at points, or they group together along lines. The zero-dimensional discontinuities, at points, indicate singularities, where orientation preferences change by more than $90^{\circ}$ between pixels, and which arise at the centers of vortices where orientation preferences rotate continuously through $\pm 180^{\circ}$. Short one-dimensional lines indicate fractures, where preferences change discontinuously by less than $90^{\circ}$, and which run between adjacent regions of cortex where orientation preferences change linearly. 

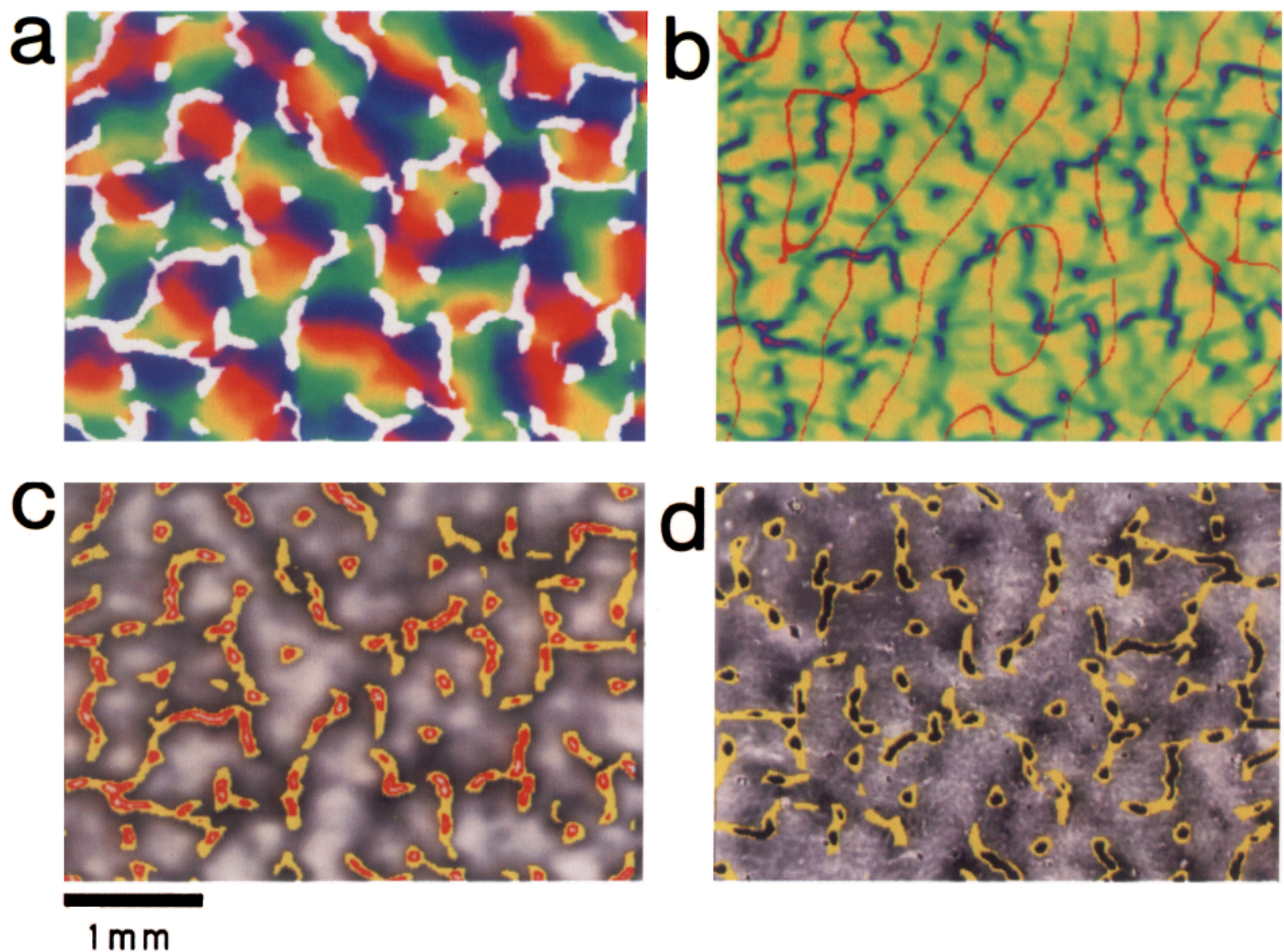

$1 \mathrm{~mm}$

Figure 8. These images show the relationship between the gradient discontinuities in Figure $7 c$ and other features of cortical organization. $a$, Discrete gradient values exceeding $45^{\circ}$ (between pixels) are indicated against the map of orientation preferences (Fig. $7 a$ ). From this it is clear that lines of sharp change extend between conflicting areas on either side that spread cohesively for some distance. $b$, While the precise relationship between ocular dominance and orientation remains to be determined, discontinuous events clearly predominate in the centers of ocular dominance bands (Blasdel and Salama, 1986), as one can see easily in this visual overlay, where the edges of ocular dominance bands (derived from zerocrossings) are indicated against a reverse-contrast image of the gradient (discontinuous changes are indicated by dark regions). Note that fractures running parallel to the ocular dominance bands tend to lie in their centers, and frequently contain singularities. Fractures running perpendicular to the ocular dominance bands (diverging by more than $45^{\circ}$ ), on the other hand, tend to be located at their edges. Both of these tendencies are particularly apparent in Figure 9, where discrete gradient values appear separately through windows corresponding to the centers and edges of ocular dominance bands. $c$, In this image, fractures have been added to the map of orientation selectivity (Fig. $7 b$ ). The high apparent correlation is expected from the likelihood of lateral inhibitory enhancements that might sharpen orientation selectivity, as well as from previous observations that orientation selectivity is reduced near blobs (Livingstone and Hubel, 1984a), since these are correlated with fractures (see below). Though it is likely to be valid, one should be cautious in accepting this correlation on the basis of optical data alone, since the apparent selectivity could be influenced by proximity to fractures if there is any lateral mixing of optical signals (through light scatter, defocused images above and below the plane of focus, lateral averaging, etc.), which cannot be ruled out at present. $d$. This figure shows a visual overlay of fractures and cytochrome oxidase blobs. Even though the image of cytochrome oxidase-stained tissue has been photographed under the same microscope, expanded, and realigned (through warping) with respect to vertically running arterioles, there are inescapable problems with alignment resulting from uncertainties about depth (from which the differential images and cytochrome oxidase-stained sections were obtained) and parallax. Nevertheless, fractures are clearly more abundant near regions of elevated cytochrome oxidase activity, as can be seen from the discrete gradient values that are $34 \%$ higher (with $46 \%$ greater fluctuations) in the darker, cytochrome-dense portions of this image (Table 1).

that of linear and nonlinear zones. And this suggests a correspondence between linearity and selectivity that is underscored in Figure $8 c$, where discontinuous shifts in preference are indicated over a map of orientation selectivity. As one can see in this overlay, singularities and fractures populate the darker zones, avoiding the lighter ones altogether.

Since fractures and singularities correlate with regions of elevated cytochrome oxidase activity (see below), the relation between linearity and selectivity is consistent with previous reports of reduced selectivity near blobs (Livingstone and Hubel, 1984). The correspondence is inexact, however, since fractures run between blobs as well. The correlation between linearity and selectivity (Fig. $8 c$ ) suggests, therefore, that the reduced orientation selectivity near blobs reflects reduced selectivity in more extensive regions that include blobs.

\section{Ocular dominance and orientation}

When Hubel and Wiesel $(1972,1974 a)$ proposed that orientation preferences are organized in slabs, they suggested the possibility of a simple geometric relationship, that slabs of orientation and ocular dominance might intersect at consistent angles. This was tested later on, where it appeared not to fit observations 

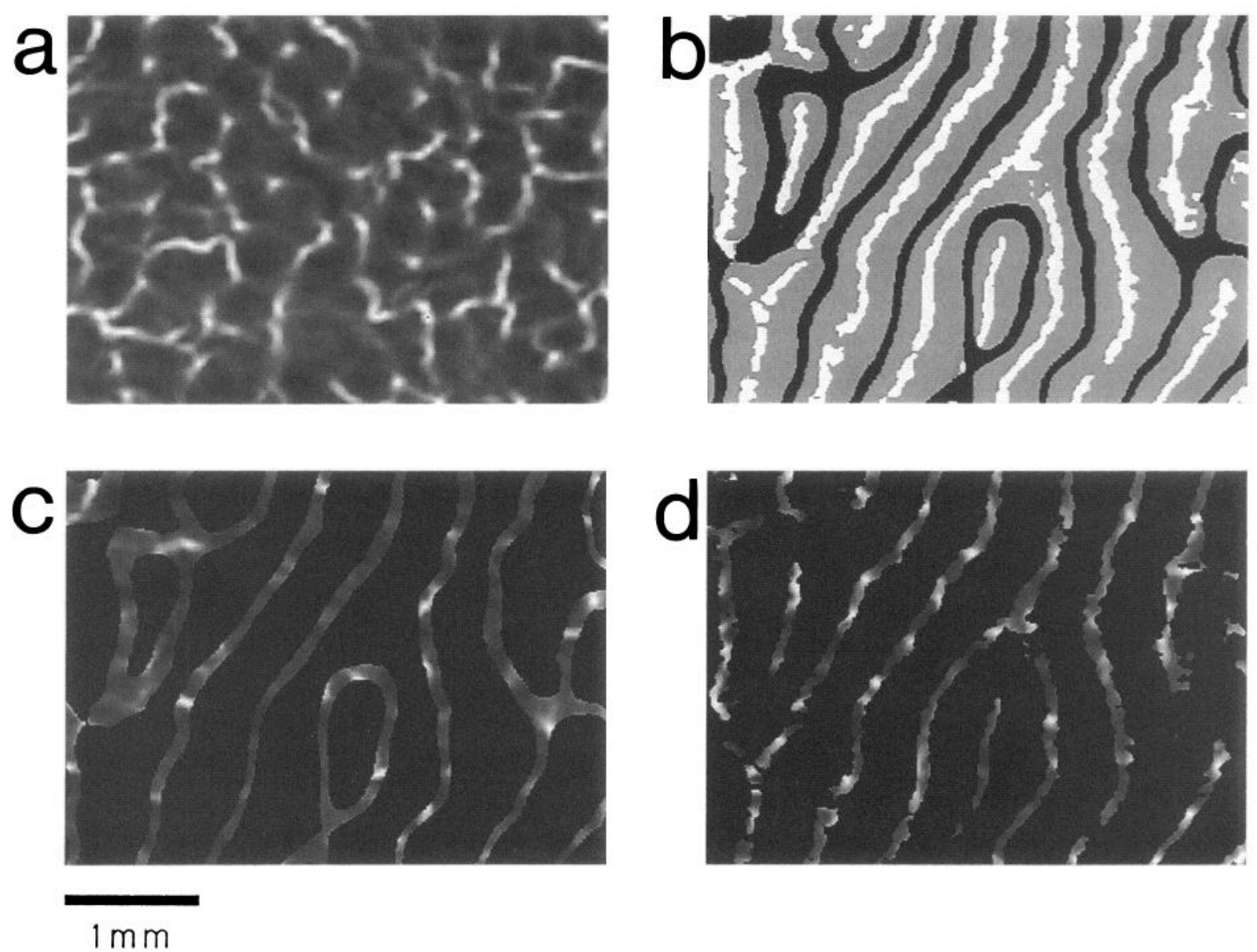

$1 \mathrm{~mm}$

Figure 9. Correspondence between ocular dominance bands and fractures. For convenience, the gradient pattern in Figure $8 a$ is replicated in $a$. In $b$, one can see the template used to isolate the centers and edges of ocular dominance bands (for a detailed explanation, please refer to the preceding article, Blasdel, 1992). The patterns in $c$ and $d$ show the gradient disruptions through windows exposing only the edges $(c)$ and centers (d) of the ocular dominance bands. As one can see, they are more abundant in the centers. From $a$, one can also appreciate that the few disruptions apparent at the edges are produced by fractures running transversely. This striking difference can also be shown quantitatively because discrete gradient values in the centers have a mean and standard deviation that are $55 \%$ higher than they are at the edges (see Table 1 ).

that 2DG-labeled "orientation" bands intersect ocular dominance bands at all angles (Hubel et al., 1978). Since it is now clear that bands of $2 \mathrm{DG}$ label do not indicate preferences for single orientations (Horton and Hubel, 1980; Blasdel, 1992), the possibility of a relationship still exists.

The first direct evidence for such a relationship came from the tendency of fractures to align with the centers of ocular dominance columns (Blasdel and Salama, 1986). This is evident in Figure $8 b$, where lines corresponding to ocular dominance boundaries have been added to a reverse-contrast image of the gradient. The disruptions indicated by dark values are clearly more abundant in the centers of ocular dominance-between the lines-than they are at the edges. Where these disruptions extend as fractures, they clearly are aligned more parallel to the ocular dominance columns in their centers, and more perpendicular to the ocular dominance columns at their edges. On account of both tendencies, regions between the centers of adjacent ocular dominance columns are the least likely to be marred by sudden transitions. These linear zones are also positioned to receive the most balanced binocular input.

The correlation between disruptions and ocular dominance is particularly evident in Figure 9, where portions of the gradient appear separately through windows corresponding to the edges
(Fig. 9c) and centers (Fig. 9d) of ocular dominance bands. The greater abundance of disruptions in the centers can also be shown quantitatively, by calculating mean rates of change. In the centers of ocular dominance columns (Fig. 9d), these rates are $55 \%$ higher than they are at the edges (Fig. 9c; see also Table 1). Since the standard deviation is also greater in the centers, by $56 \%$, this difference reflects a greater number of disruptions rather than generally elevated values. One might note, moreover, that this calculation ignores the alignment between fractures and ocular dominance, which, if taken into account, would amplify the difference.

\section{Fractures and cytochrome oxidase}

Disruptions in continuity and cytochrome oxidase blobs are both concentrated in the centers of ocular dominance columns, so they should be correlated. This is apparent in Figure $8 d$, where abrupt changes are indicated on an aligned image of cytochrome oxidase-stained tissue. It can also be shown quantitatively from the fact that orientation preferences change $38 \%$ more abruptly, with $46 \%$ greater fluctuations, in cytochrome oxidase-rich regions (see Table 1). The exact relationship is complicated, however, since fractures also run between blobs, and since singularities usually do not lie in their centers. 


\section{Singularities}

As pointed out above, the continuity of orientation preferences is interrupted semiperiodically by fractures and singularities. Fractures extend along lines, because they entail jumps of $90^{\circ}$ or less, but they themselves are interrupted at points where the gradient exceeds $90^{\circ}$. Shifts exceeding $90^{\circ}$ cannot cluster along lines because the greatest shift possible, in the orthogonal direction, is only $90^{\circ}$, but shifts exceeding $90^{\circ}$ can occur at points, in two dimensions, if orientation preferences rotate continuously all around. If they rotate continuously through $\pm 180^{\circ}$ in one revolution, for example, those on opposite sides are always perpendicular (see Fig. 1 1) such that $\Delta \theta / \Delta x$ and $\Delta \theta / \Delta y$ are both $90^{\circ}$, and the gradient in the middle approaches $\sim 127^{\circ}$. Such points are singularities bccausc surrounding trends cannot bc resolved in any other way. Since orientation preference is undefined at singularities, a loss of orientation selectivity is required.

The tendency for orientation preferences to form singularities is obvious in Figure 10, $a$ and $b$, where preferences and selectivities are indicated by the orientations and lengths of short lines. Figure $10 a$ shows the relationship between these lines and the color-coded map in Figure $7 a$, while Figure $10 b$ shows the relationship with the gradient. From the latter, it is obvious that the darker spots, denoting singularities, arise in the centers of vortices where orientation preferences rotate continuously (along some paths) through $\pm 180^{\circ}$. Because they cycle through $180^{\circ}$ (and not $360^{\circ}$ ), the topology of orientation preferences conforms to a ridge system rather than vectors (Penrose, 1979). Because there are no directional components, as there are with vectors, oricntation preferences rotated through $180^{\circ}$ are equivalent.

For any continuous path around a singularity, orientation preferences change in either the same or the opposite direction. For example, if we assume that paths are always taken in a positive (counterclockwise) direction, cumulative rotations through $180^{\circ}$, in a positive (counterclockwise) direction, reflect a positive singularity, with a value of +1 , while cumulative rotations through $-180^{\circ}$, in a negative (clockwise) direction, reflect a negative singularity, with a value of -1 . From Figure 11 , it is clear that positive singularities form "loops" while negative singularities form "tri-radii."

From principles governing the topology of ridge systems in two dimensions (Penrose, 1979), one can show that singularities can be added to infer the cumulative rotation around all of them. Hence, a continuous path around two positive singularities encounters a net rotation through $360^{\circ}(+2)$, while a continuous path around onc positive and one negative singularity encounters a net rotation through $0^{\circ}$. These principles make it possible to reach several conclusions. The first, which has already been mentioned, is that positive "loop" and negative "tri-radius" types of singularities must be real since they derive from welldefined orientation preferences all around; they cannot be produced by any artifacts in the space enclosed (e.g., by reduced selectivity near blobs). The second is that positive and negative singularities must be present in equal numbers since closed paths, of arbitrary diameter, encounter cumulative rotations of 0 or \pm 1 revolution (in Figs. $6 a, c ; 7 a$ ) as long as they are taken through zones of continuous change.

\section{Linearity of change versus density of orientations preferred}

From Figure 7, $a$ and $c$, it is evident that most of the cortical surface is occupied by linear zones, and that orientation pref-
Table 1.

\begin{tabular}{llllll} 
& \multicolumn{5}{l}{$\begin{array}{l}\text { Orientation gradient } \\
\text { (degrees of shift/pixel) }\end{array}$} \\
\cline { 2 - 6 } Pattern & $X_{m}$ & rms & $N$ & $\Delta X_{m} / X_{m}$ & $\begin{array}{l}\Delta \mathrm{rms} / \\
\text { rms }\end{array}$ \\
\hline Sample 1 & & & & & \\
$\quad$ Centers & 17 & 14 & 1273 & $55 \%$ & $56 \%$ \\
$\quad$ Boundaries & 11 & 9 & 1518 & - & - \\
Sample 2 & & & & & \\
$\quad$ Centers & 17 & 14 & 2647 & $55 \%$ & $40 \%$ \\
$\quad$ Boundaries & 11 & 10 & 3408 & - & - \\
Sample 3 & & & & & \\
$\quad$ Blob & 18 & 19 & 2167 & $38 \%$ & $46 \%$ \\
Interblob & 13 & 13 & 8949 & - & - \\
\hline
\end{tabular}

Samples 1 and 2 show the correlation between the mean rate $\left(X_{m}\right)$ at which orientation preferences change between pixels and ocular dominance. Mean rates were calculated from the formula $X_{m}=(\Sigma X) / N$, where $N$ equals the number of values in the sample area. The root mean square (rms) was calculated with the formula mis $=\left[\Sigma_{i-1 . N}\left(X_{i}-X_{m}\right)^{2} /(N-1)\right]^{0.5}$, and $\Delta X_{m} / X_{m}$ was determined from $\left(X_{\text {m(center) }}-X_{\text {m(thoundary }}\right) / X_{\text {m(therundary }}$. In sample 1 , the mean rate was calculated (for both the boundaries and centers of ocular dominance bands) from values appearing in the central portions of Figure 9, $c$ and $d$. In samplc 2, the same calculations were made from all the values in Figure 9, $c$ and $d$. As one can see, $X_{m}$ is $55 \%$ higher in the centers of ocular dominance bands, and because rms values are also higher, by $56 \%$, this results from a greater number of disruptions rather than a uniformly higher rate. Sample 3 shows the relation between mean rates of change and levels of cytochrome oxidase activity. $X_{m}$ and rms values are both higher in zones exhihiting high cytochrome oxidase activity (blob), which were taken as those that were $80 \%-100 \%$ as dark as the darkest regions, after histochemical staining for cytochrome oxidase (Wong-Riley, 1979).

erences are represented at different densities in different areas. This is because they require $\approx 700 \mu \mathrm{m}$ to complete a $180^{\circ}$ rotation in linear zones, and much less near singularities and fractures. One consequence of this is a large variation in the range of orientation preferences available to cells. If one assumes the dendritic fields of upper-layer neurons are approximately cylindrical, $300 \mu \mathrm{m}$ across (Lund and Yoshioka, 1991), the proportion of possible orientation preferences received will vary between $100 \%$, near singularities, and $40 \%$ or less in the linear zones. (Even though this calculation emphasizes orientation preferences, ignoring ranges of response, the consequences would be similar if selectivities were taken into account since orientation selectivity is highest in the linear zones, as indicated in Fig. 8c.)

These variations are emphasized in Figure $10, c$ and $d$, where regions showing the highest densities and linearities of orientation preference are depicted separately. From these two representations, it is clear that there is a tradeoff between linearity and density in the mapping of orientation preferences, and that each must be optimized separately. One might also note that, even though linear zones occupy most of the cortical surface (Fig. 10d), that the range of orientation preferences represented (per square millimeter), is approximately the same in Figure $10 c$ where density is optimized instead.

\section{Geometry of orientation and ocular dominance slabs}

Since 2DG-labeled "orientation columns" are ambiguous with respect to orientation (Blasdel, 1992), there is still a possibility that ocular dominance and orientation slabs intersect at consistent angles (Hubel and Wiesel, 1974a). This was evaluated recently (Obermayer et al., 1992) by using Gabor-Helstrom transforms to measure orientation and ocular dominance trends 

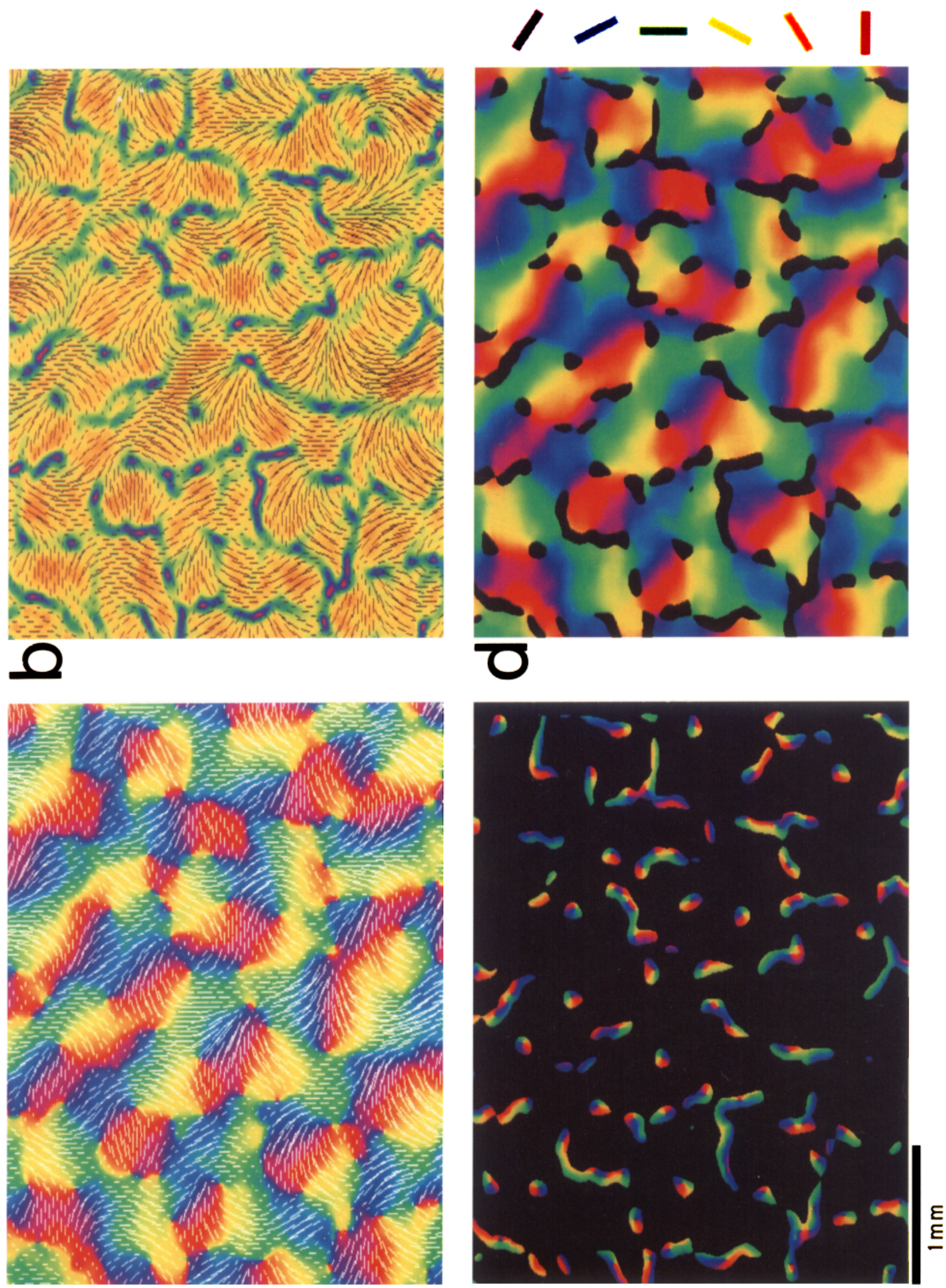

ธ

0 
a

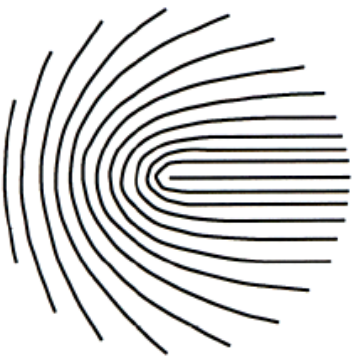

b

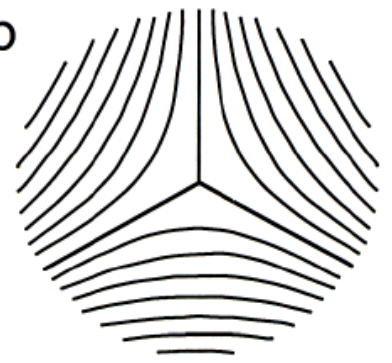

Figure 11. Singularities in Ridge systems (Penrose, 1979). Because orientation preferences are not directed, ones rotated through $180^{\circ}$ are equivalent. They generate a singularity therefore when they rotate continuously through $\pm 180^{\circ}$ around a point. $a$, When they rotate in the same direction, as the path taken around the point, they form a positive singularity, with a value of +1 and a characteristic looplike structure. $b$. When they rotate in the opposite direction, they form a negative singularity, with a value of -1 and a tri-radius-like structure. Both loops and tri-radii can be identified around singularities, in the patterns of short lines in Figure 10, $a$ and $b$. After Penrose, 1979.

at 3424 locations, and showing that where orientation preferences are mapped most linearly, orientation and ocular dominance slabs project in orthogonal directions. This relationship can also be seen in Figure 12a, where ocular dominance centers have been occluded. As one can see, this highlights the isoorientation slabs by covering the singularities (which are highlighted reciprocally in Fig. $12 b$ ), revealing a strong orthogonal relationship between the centers of ocular dominance columns and the shorter iso-orientation slabs that project perpendicularly in between.

\section{Reliability and artifacts}

Reliability. One would like to verify that the procedures illustrated in Figure 2 are sound. Since the major assumption was linearity, the simplest approach is to verify that the maps of orientation preference and selectivity in Figure 7, $a$ and $b$, are linear superpositions of the differential images used as input. If they are, the operations illustrated in Figure 2 should operate in reverse; the cosine and sine components of output vectors should reflect preferences for vertical/horizontal and left/right oblique similar to those of the differential images (for vertical/ horizontal and left/right oblique) that were acquired initially
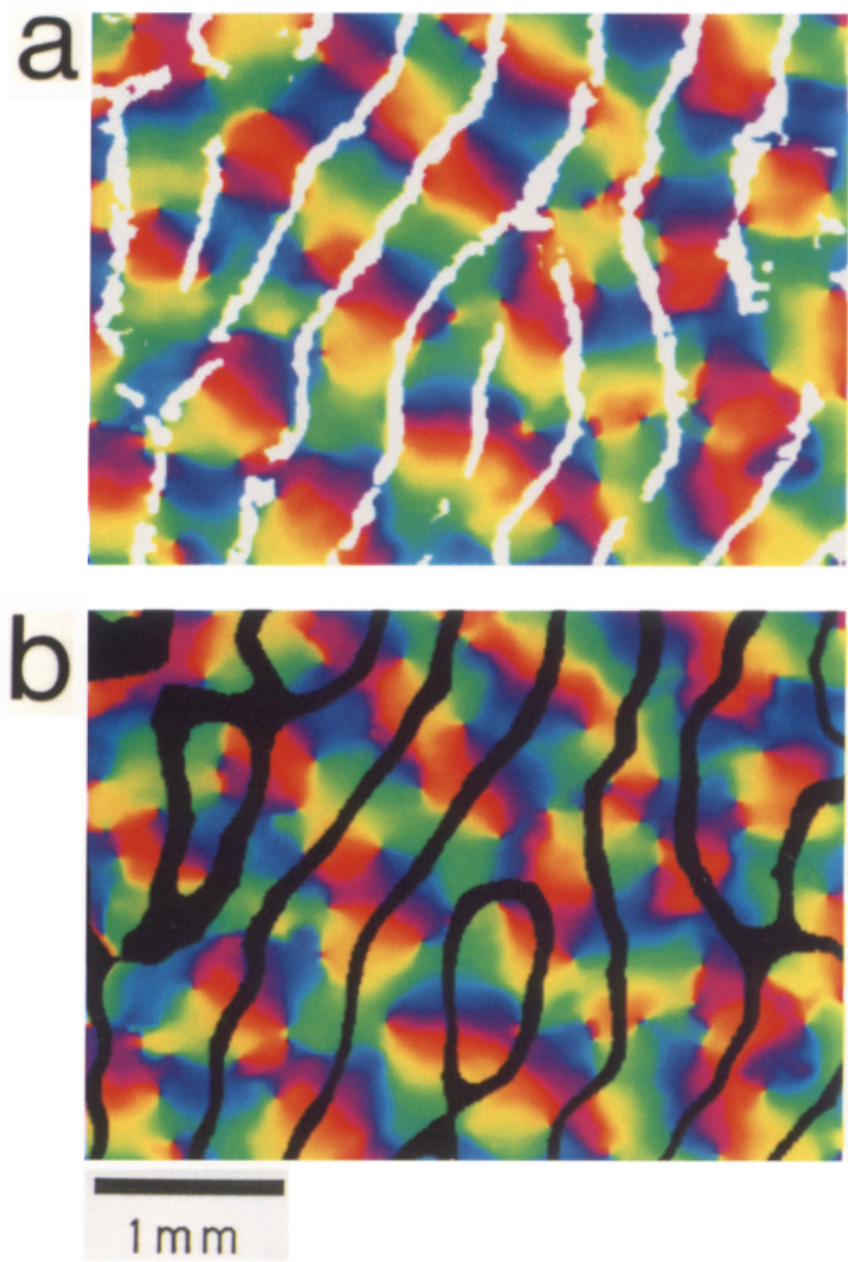

Figure 12. Geometric relationship between ocular dominance and orientation slabs. The centers and edges of ocular dominance bands, from the template in Figure $9 b$, are indicated over the map of orientation preferences, from Figure $7 a$, in $a$ and $b$. As one can see, the regions where orientation slabs run parallel extend between singularities in the centers of adjacent ocular dominance bands, which are whited out in $a$ but obvious in $b$. This gives them an orthogonal relationship $\left( \pm 45^{\circ}\right)$ with respect to the ocular dominance slabs, as suggested originally by Hubel and Wiesel (1974a). As a consequence, orientation preferences have a marked tendency to rotate linearly in a direction parallel to the ocular dominance slabs, and to remain constant in the perpendicular direction where it is proposed that retinotopic positions might change linearly instead (see Results).

\footnotetext{
Figure 10. Positive and negative singularities are easier to appreciate when orientation preferences are indicated by short, oriented lines. $a$, The correspondence of orientation with color can be seen in this figure, where it also should be apparent that orientation selectivity can be indicated as well, by the length of each line. $b$, Overlay of short oriented lines (indicating orientation preference and selectivity) with an inverse-contrast image of the gradient (i.e., dark values indicate regions of discontinuous change). Note that lines tend to be longer (indicating high selectivity) in the continuous regions, and that more orientations per unit area are represented near regions of discontinuous change (indicated by dark spots and lines). Singularities are apparent from the gradient as dark spots. As one can see from the short oriented lines, these are regions where all orientation preferences converge. On account of the continuity outside singularities (and associated fractures), the preferred orientation around each one cycles through $180^{\circ}$ along any closed path. It does so in either a positive or a negative direction (which, for a counterclockwise path, means a counterclockwise or a clockwise rotation). These are apparent (in the contours formed by short line segments) as "loops" and "tri-radii" (see Fig. 11), which, as one can see, are roughly balanced. In addition to bringing all possible orientation preferences together, within the smallest conceivable radii, both organizations ensure that perpendicular orientations are always represented on opposite sides. These are the only places in cortex where all possible orientation preferences are accessible within the dendritic fields of "typical" upper-layer cells, that is, within radii of less than $250-350 \mu \mathrm{m}$. $c$, Distribution of orientation preferences where they are changing quickly, about singularities and fractures. Note that, even though the indicated regions cover much less area than those in $d$, all orientation preferences are nevertheless present in each part of the visual field due to a much higher density. Note also that all orientation preferences are brought uniquely close together, around points corresponding to the singularities, and along lines corresponding to the fractures. These are the only regions where representations for all orientations converge to areas smaller than those sampled by single dendritic fields, $250-350 \mu \mathrm{m}$ in diameter (Lund and Yoshioka, 1991). $d$, Distribution of orientation preferences in the continuous patches, where they change linearly but slowly. Regions of rapid change, corresponding to the indicated areas in $c$, have been blacked out. Even though orientation is defined precisely, and linearly, representations of all orientations cover large areas, at least $700 \mu \mathrm{m}$ across. Consequently, the neurons in these zones, with average $250-350-\mu \mathrm{m}$-wide dendritic fields, would receive information over a range of only $\sim 60^{\circ}$.
} 
a

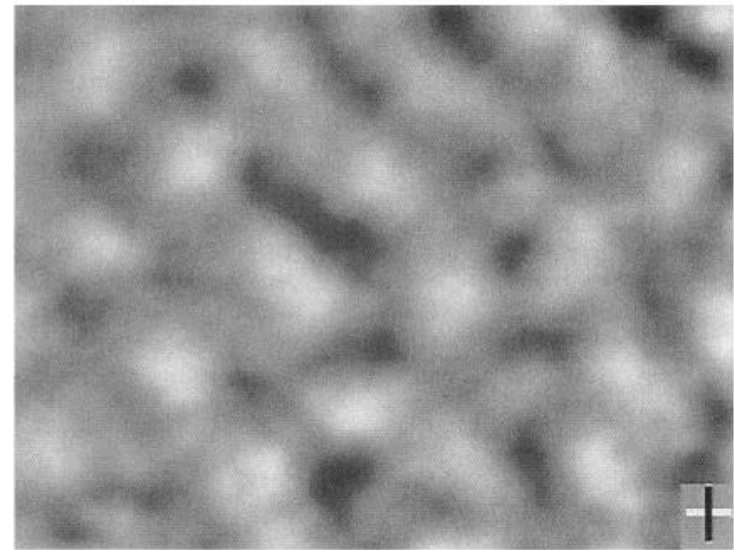

\section{C}

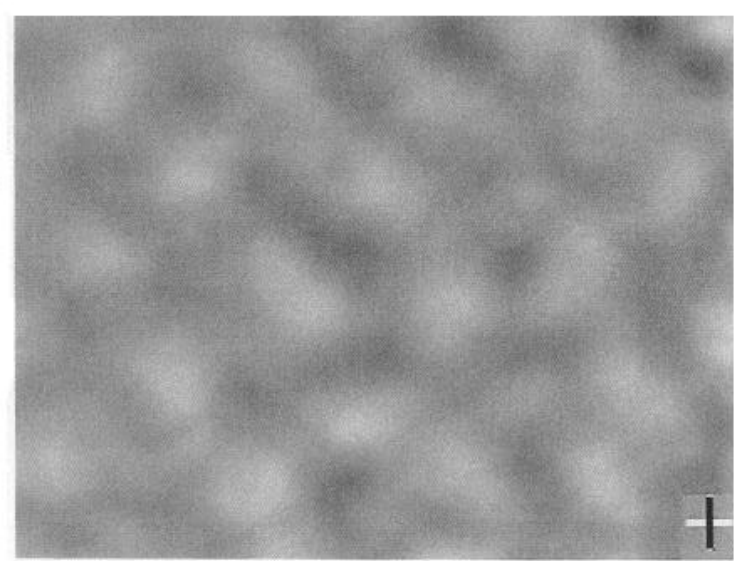

e

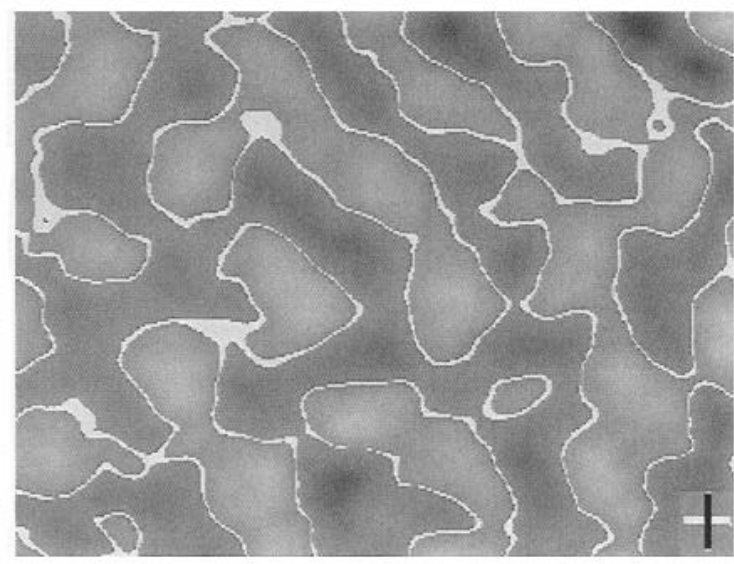

$1 \mathrm{~mm}$ b

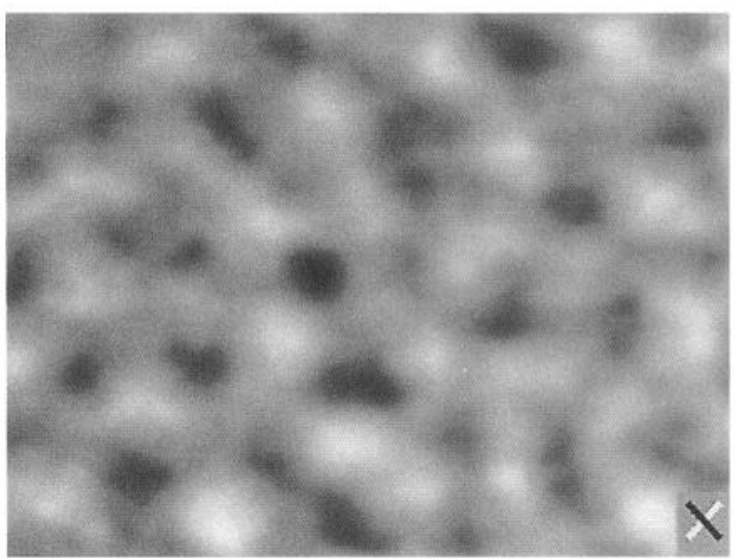

d

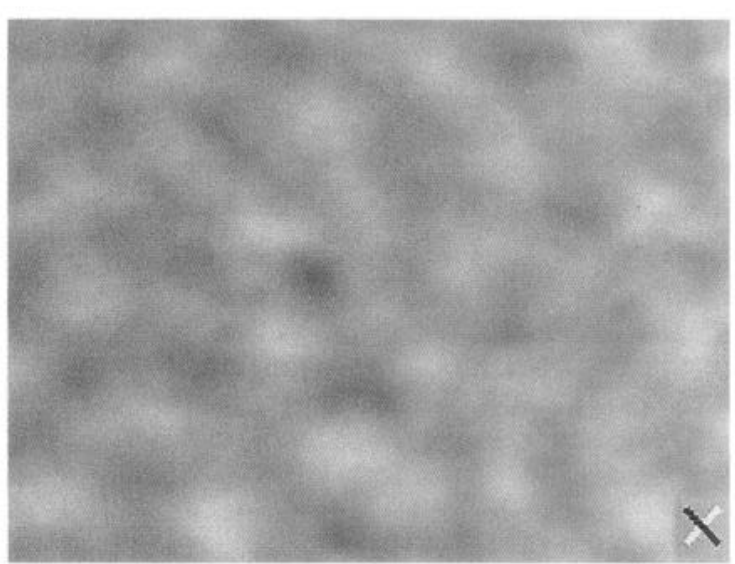

f

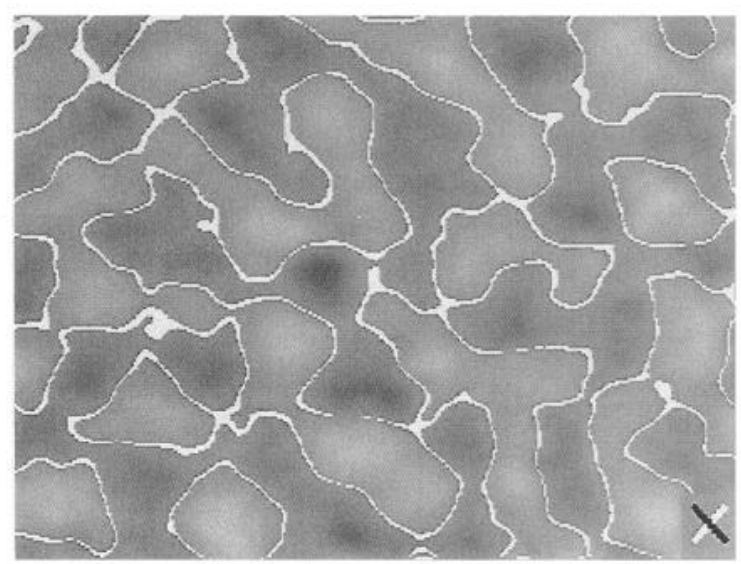

Figure 13. The images in $a$ and $b$ show the vectors in Figure 4 in Cartesian (rather than Polar) coordinates where images of $\mathrm{x}$ - and $\mathrm{y}$-components should correspond to preferences for vertical/horizontal and left/right oblique, respectively. The initially acquired differential images of vertical/ horizontal and left/right oblique are provided below in $c$ and $d$ for comparison. As one can see in $e$ and $f$, where zero-crossings from the images in $a$ and $b$ have been added to the images in $c$ and $d$, these patterns are virtually identical, with the only obvious difference being that those in $a$ and $b$ exhibit higher signal: noise ratios than those in $c$ and $d$ on account of greater averaging.

Figure 14. Effects of blood vessels on selectivity and continuity. $a$, An image of the vasculature under green light (chosen to maximize contrast with the blood vessels). $b$, An outline of prominent blood vessels (those most likely to interfere with light transmission). $c$, Outline of prominent blood vessels from $b$, superimposed on the image of orientation selectivity from Figure $7 b$. As one can see from this superposition, blood vessels are not correlated with zones of reduced selectivity, except possibly at the intersection of two large ones (which would be discounted in any case). 

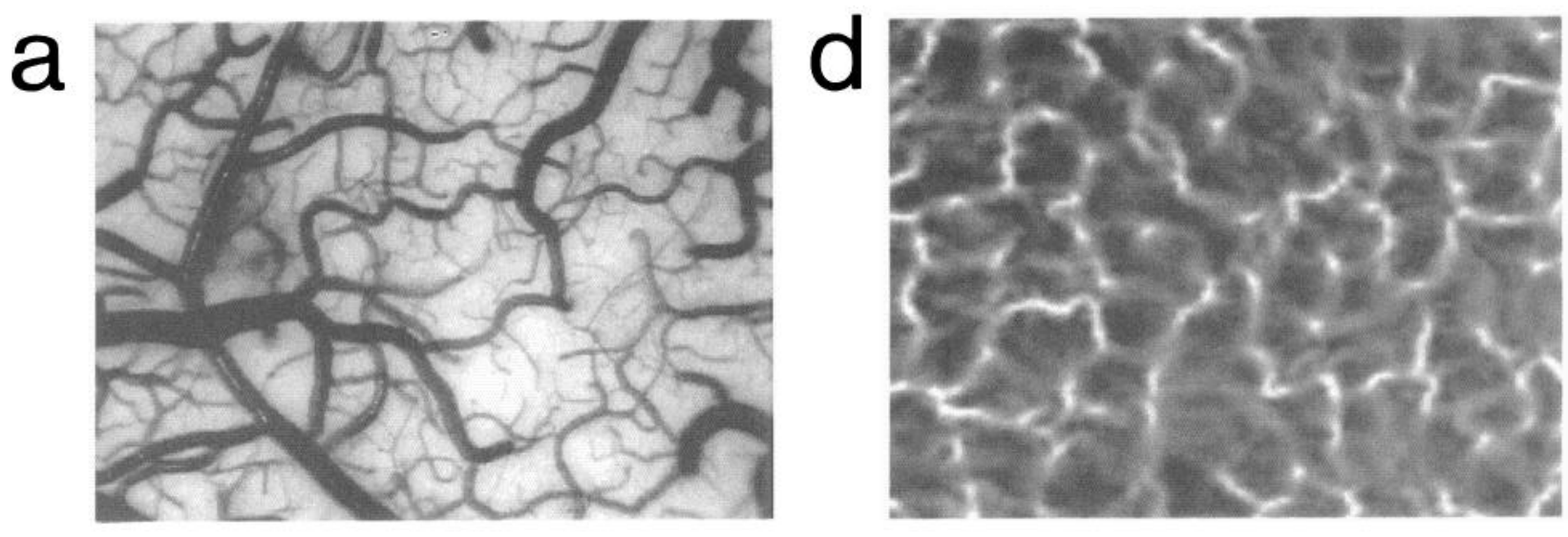

b
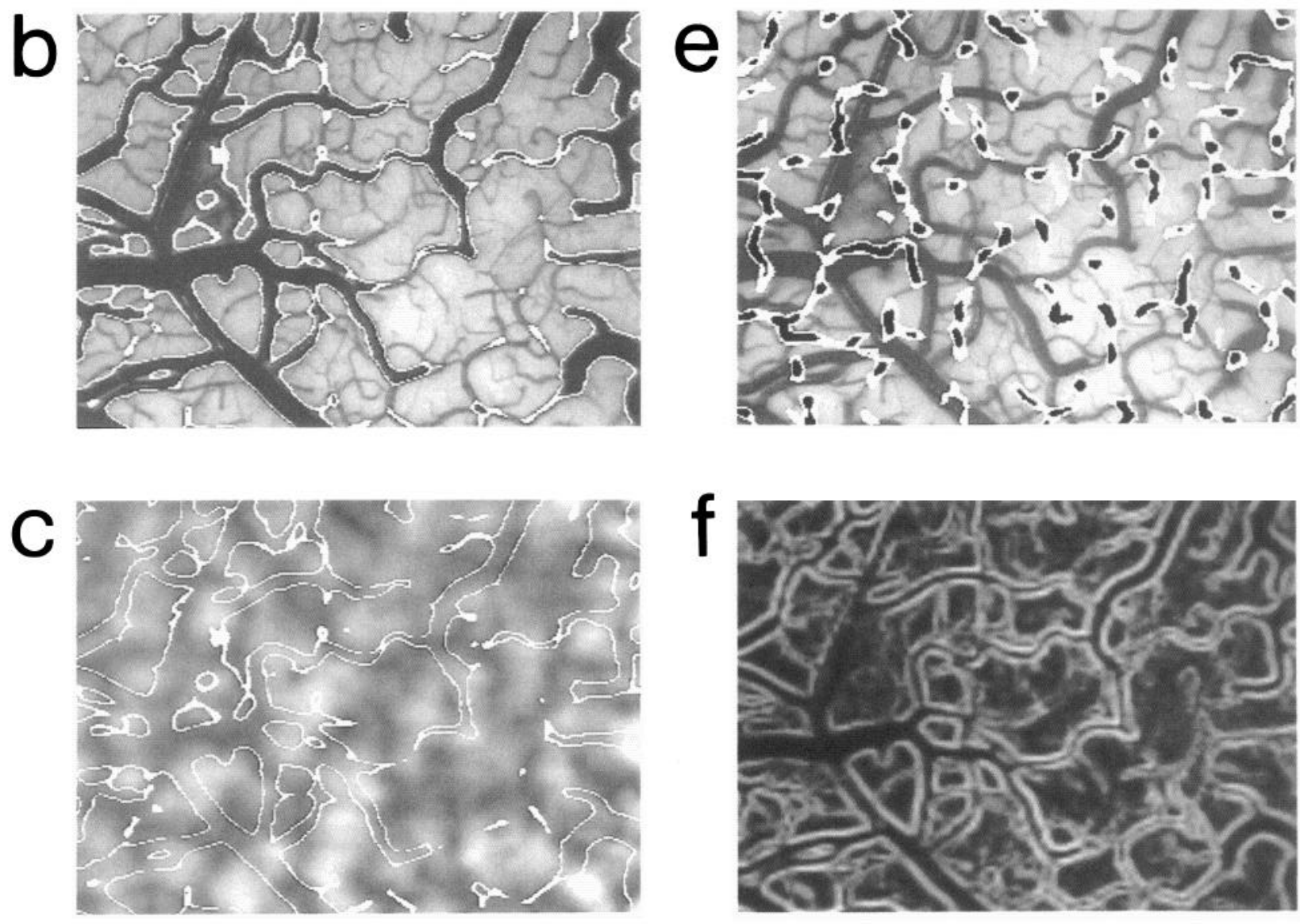

\section{$1 \mathrm{~mm}$}

Medium-sized blood vessels pass just as frequently over regions of high selectivity as they do over regions of low selectivity. And even in the latter case, where they do pass temporarily over zones of reduced selectivity, they are rarely aligned. $d$, Orientation gradient, duplicated from Figure $7 c$ for comparison. In spite of a vague similarity in shape, fractures differ strikingly from blood vessels in distribution, as one can see by comparing this image with that in $a$. Differences are manifest in the way that blood vessels frequently cross one another, while fractures never do, as well as in the fractal distribution of blood vessels-similar patterns are apparent at different scales-which contrasts strongly with the uniform size and semiperiodic distribution of fractures. $e$, This overlay reveals the positions of discontinuities exceeding $45 \%$ pixel in relation to the vasculature. As one can see, however, there is no correlation. Fractures occur just as frequently in relatively avascular regions of cortex as they do in ones with many large blood vessels. Those that do occur near blood vessels are just as likely to intersect them at right angles as they are to run parallel. And while there is a hint that one of the largest blood vessels (center left) might have induced a fracture, this is unlikely for two reasons: (1) the fracture in question consists only of one line, and (2) it can be seen in Figure $8 a$ to lie between two continuous areas with decidedly different orientation preferences extending cohesively (beyond the blood vessel) on either side. $f$. Apart from the absence of correlation between blood vessels and fractures, the best evidence that they are not related is that real vascular artifacts look much different. The line of disturbance along a blood vessel (see Fig. 15a) might displace values momentarily, along a line, but these have to be resolved on either side. Accordingly, the artifacts from blood vessels occur in pairs-one on either side-examples of which can be seen clearly in this image, which was produced by taking a gradient of the vascular image in $a$ directly. 
a
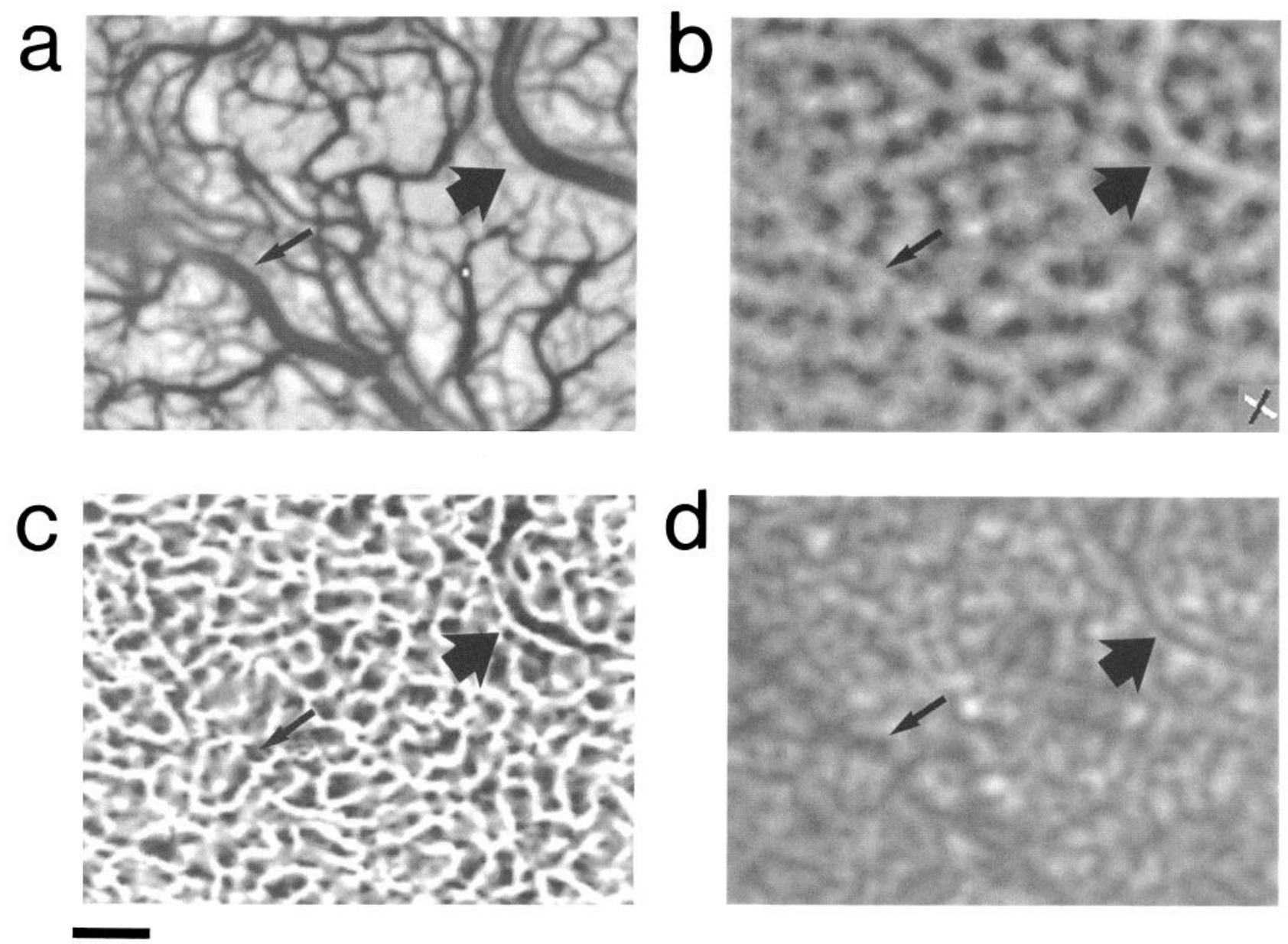

$1 \mathrm{~mm}$

Figure 15. The freedom from vascular interference of most images is easily appreciated from the occurrence of real vascular artifacts, which are not subtle. $a$, The vascular pattern covering the cortex from which orientation preferences were imaged in Figures 3 and 6 . $b$, One of the initially acquired differential images, where two unmistakable artifacts (arrows) have clearly been induced by large blood vessels. One normally tries to reduce such noise (by adjusting the tidal volume, respiration rate, duration of frame collection, drugs, etc.), but their complete elimination is not always possible, especially with blood vessels larger than $200 \mu \mathrm{m}$ in diameter. The smaller and larger blood vessels (arrows) leading to artifacts in these images are $290 \mu \mathrm{m}$ and $360 \mu \mathrm{m}$ in diameter, respectively. The resulting artifacts can nevertheless be recognized and excluded from further analysis. $c$, Clear and unmistakable artifacts are apparent in the orientation gradient as well, as one can appreciate from this image. The artifact in the upper right is apparent as a pair of lines, as suggested by Figure 14f. And even though the one on the left is broken along its length, there clearly is something unusual along the path covered by the blood vessel. $d$. From this map of orientation selectivity one can see that blood vessels larger than $200 \mu \mathrm{m}$ in diameter actually can block light, and reduce the apparent selectivity for orientation. It is important to realize, however, that these effects are not subtle. When they occur, vascular artifacts are obvious in all stages of processing, from the first differential images, where they are recognized easily (and avoided) on account of their elongated shapes and close associations with blood vessels.

and used as part of the input. If they match, one can conclude (1) that the operations in Figure 2 operate in reverse, (2) that differential images of orientation (Fig. 5) add nondestructively when normalized for orientation, (3) that positions of maxima and minima in each differential image are linear functions of stimulus orientation, and (4) that the operations in Figure 2 are therefore unlikely to have distorted the data.

This test is performed in Figure 13, where $a$ and $b$ show cosine and sine coefficients for the vectors displayed (in Polar coordinates) in Figure 7, $a$ and $b$. These should reflect preferences for vertical/horizontal and left/right oblique. And, as one can see by comparing them with actual differential images in Figure 13, $c$ and $d$, the correspondence is close. Dark and light peaks occupy similar locations, as do boundaries in between. The obvious alignment is emphasized in Figure 13, $e$ and $f$, where zero-crossings from Figure 13, $a$ and $b$, have been added, making it clear that the main difference (between Fig. 13, $a, b$ and $c, d$ ) arises from greater signal : noise ratios in Figure 13, $a$ and $b$, which reflect contributions from many more images.

Vascular artifacts and fractures. Vascular artifacts were also considered extensively in the preceding article (Blasdel, 1992). The exercise was largely academic, however, since there was no obvious reason for suspecting blood vessels in the first place: they are virtually transparent to light at $720 \mathrm{~nm}$ (the wavelength used to monitor activity), and they do not resemble differentially imaged patterns at all. Even subtle influences could usually be ruled out. This is more difficult to argue in the case of fractures, however. Even though we have no reason to assume that vascular artifacts have reemerged spontaneously, the superficial similarity between fractures and blood vessels has led to some speculation that the two are related (Grinvald et al., 1986; Orbach and Van Essen, 1986). 
Figure 14, $a$ and $d$, shows vascular and fracture patterns from the same region of cortex where, despite superficial similarities, they clearly differ. While blood vessels appear in a variety of sizes (Fig. 14a), with self-similar distributions across scales (i.e., they are fractal), fractures always consist of isolated lines, distributed periodically at one scale. Another difference is that blood vessels cross one another frequently while fractures never do. The visual overlay of fractures and blood vessels in Figure $14 e$ makes these differences obvious. Fractures extend just as frequently over lightly vascularized regions as they do over heavily vascularized ones. And even when they occur in the latter regions, they are just as likely to cross blood vessels at right angles as they are to run along one side. They usually are not aligned.

One therefore should consider whether fractures could be generated by vascular artifacts. A singularity cannot be explained in this way because it reflects trends in orientation preference all around-trends that extend well beyond the boundaries of any particular blood vessel. The singularity is required by these trends, whether it overlaps with a blood vessel or not. The same is true for a fracture, which divides separate trends extending continuously on either side for some distance.

Another way of addressing this issue is to consider what a real vascular artifact might look like. The discontinuities in a vascular image, for example, have a different distribution. These are depicted in Figure 14f, where the resulting lines clearly look like blood vessels. And, unlike the linear discontinuities in Figure $14 d$, the discontinuities in Figure $14 f$ extend in pairs-one on either side of each blood vessel producing them-as one would expect.

An actual artifact, arising from a blood vessel too large to be avoided, appears in the differential image in Figure $15 b$ (large arrow), as well as in each subsequent stage of processing (Fig. $15 c, d$, as well as Fig. $6 a$ ). As one can see, this artifact generated a pair of lines (as expected from Fig. 14f). It should be emphasized that such artifacts are uncommon, however, (1) because they usually do not arise from blood vessels smaller than 150 $\mu \mathrm{m}$ (in diameter), and (2) because they are obvious when they occur. It therefore is unlikely that blood vessels contribute substantially to fractures analyzed in this article.

Artifacts and orientation selectivity. Since optically determined values of orientation selectivity are sensitive to signal intensity, there is some concern that the narrow zones of reduced selectivity (Fig. 7b) might be due to blood vessels blocking light. This can be checked by comparing values of orientation selectivity with vascular outlines in Figure $14 c$. Except in one instance, near the intersection of two large blood vessels (which would be excluded in any case), the blood vessels may be observed to follow independent trajectories, passing just as frequently over regions of high and low selectivity. And even where they overlap zones of reduced selectivity, they are not obviously aligned, as one would expect from hemoglobin's weak absorption at this wavelength $(720 \mathrm{~nm})$, as well as from the small diameters of blood vessels in this area. More interference would be expected at the shorter wavelengths used to monitor intrinsic signals (Grinvald et al., 1986; Ts'o et al., 1990).

Reduced selectivity and fractures. As noted earlier, the apparent selectivity for orientation is reduced near fractures. While consistent with previous observations (Horton and Hubel, 1980; Livingstone and Hubel, 1984a), such a correlation could also arise from other factors. One possibility is that zones of reduced selectivity reflect the lateral spread of optical signals (due to
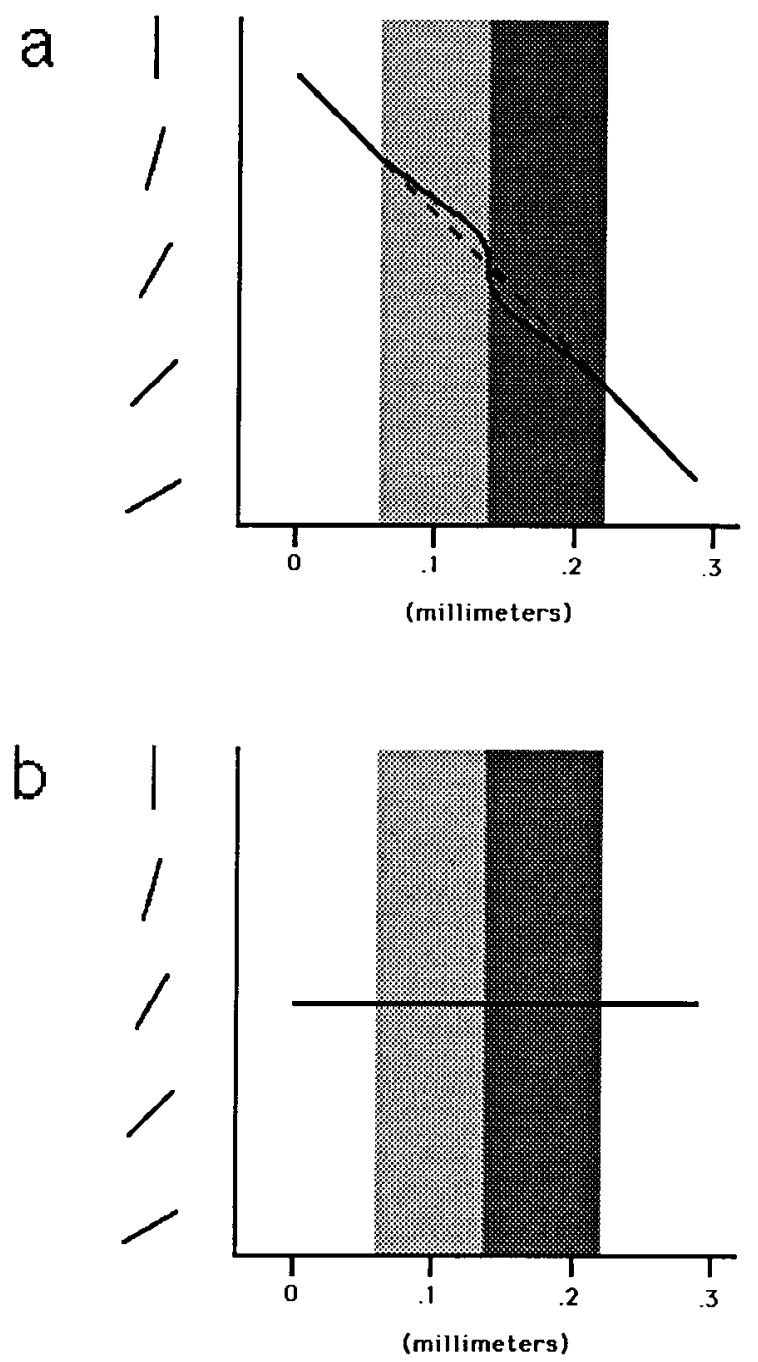

Figure 16. Illustration of one way in which an elongated region of reduced selectivity might lead to the appearance of a fracture. $a$, As one can see from this illustration, the lateral spread of signals (through light scatter, lateral averaging, etc.) across a zone of reduced selectivity (indicated by shading) might bias the apparent preferences on either side, leading to a sharp correction in the middle. Note, however, that this mechanism only works in a few cases where narrow zones of reduced selectivity run parallel to, and lie within, groups of iso-orientation slabs. When they run perpendicular, as illustrated in $b$, there is no effect because signals spread into regions preferring the same orientation. As one can see in Figure $8 a$, this is a more common arrangement: fractures and zones of reduced selectivity tend to lie at the ends of iso-orientation slabs, in the centers of ocular dominancc bands, and not at their sides, which makes the mechanism illustrated in $a$ unlikely.

averaging, light scatter, etc.) across singularities and fractures. While this is a serious concern, it cannot explain the correlation completely since reductions in orientation selectivity are also associated with blobs (Livingstone and Hubel, 1984a), which are correlated with fractures.

Another possibility is that zones of reduced selectivity somehow induce fractures (Fig. 16a). If orientation preferences really were organized in a sheet of extended slabs, an aligned fracture might be induced in the middle by a narrow channel of reduced selectivity. This would occur if there was any lateral mixing of signals (e.g., through light scatter, lateral averaging, etc.) such that stronger ones could propagate inward, invading weaker 


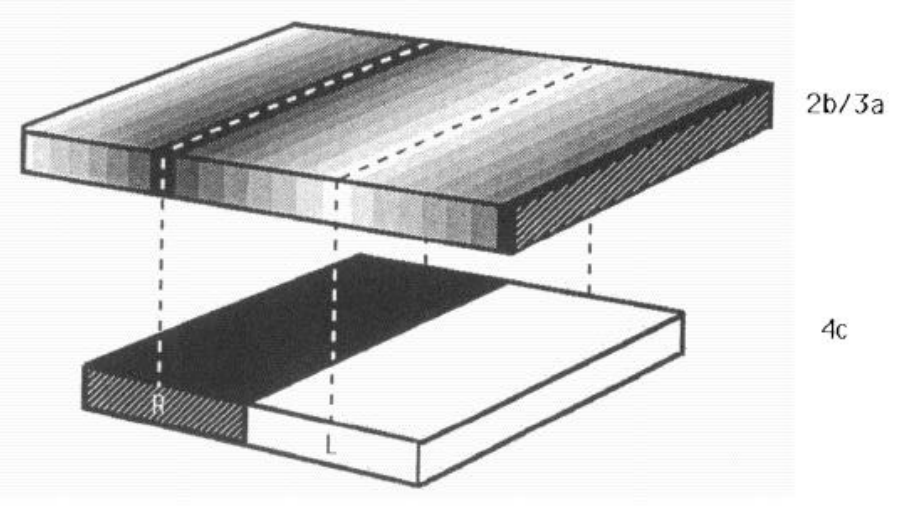

Figure 17. Binary ocular dominance slabs (e.g., Fig. 1c) exist only in layer $4 \mathrm{c}$, where geniculate afferents terminate and where cells respond only to one eye. Outside layer $4 \mathrm{c}$, where orientation selectivity emerges, binocularity also becomes important, and must therefore be illustrated separately. In Figure 16, ocular dominance is illustrated with right or left eye slabs in layer $4 \mathrm{c}$, and with many gradations of binocular slab above. From this illustration it is clear that ocular dominance shifts continuously and linearly, between extremes of right and left eye dominance, that lie over the centers of right and left eye slabs in layer $4 \mathrm{c}$. Note that this rendering places the most continuous and linear sequences of changing ocular dominance directly over the edges of ocular dominance slabs in layer $4 \mathrm{c}$, where ocular dominance changes precipitously. It also positions nonlinear reversals in the direction of change directly over the centers of ocular dominance bands in layer $4 c$, where ocular dominance is monocular but continuous.

ones from either side and thereby inducing an artificially abrupt transition in the middle.

While plausible, this mechanism requires many special conditions that usually are not present. It assumes, for example, that orientation preferences are mapped in a sheet of extended slabs, which they clearly are not since a continuous sheet cannot be reconciled with singularities. A second problem arises from attempts to explain why orientation selectivity should be attenuated in long, narrow paths. This is unlikely to occur spontaneously, and extraneous factors like blood vessels are ruled out by their lack of correspondence with fractures or zones of reduced selectivity. And lastly, as one can see in Figure $8 a$, most fractures are not aligned with iso-orientation slabs, as required by this explanation (see Fig. 16b).

\section{Discussion}

These results show that vertical/horizontal and left/right oblique components can be extracted from differential images of orientation, and averaged to infer precise maps of orientation preference and selectivity. The derived maps confirm previous observations of sequence regularity in the mapping of orientation preference (Hubel and Wiesel, 1974a), extending them to two dimensions, where the linear sequences appear as patches, and the breaks between them extend into lines.

\section{Comparison with previous work}

One surprising result is that regions preferring the same orientation extend no farther than $0.5-1.0 \mathrm{~mm}$ laterally - a distance far shorter than expected from previous observations (Hubel and Wiesel, 1974a; Hubel et al., 1978). This would have been difficult to anticipate from the results of previous recordings, since tangential electrode penetrations only sample over a few millimeters in one dimension. At this scale, the regular shifts in orientation preference are far more striking than the breaks that interrupt them, making Hubel and Wiesel's (1974a) infer- ence of parallel orientation slabs the dominant organization. But this organization breaks apart at larger scales because isoorientation slabs and linear sequences are both limited to distances shorter than $1.0 \mathrm{~mm}$.

The long bands of 2DG label that were thought to indicate "orientation columns" initially (Hubel et al., 1978) were found to contain many regions nonselective for orientation (Horton and Hubel, 1980; Humphrey and Hendrickson, 1983; Livingstone and Hubel, 1984a) and accordingly have been reinterpreted to consist of much shorter, orientation-sensitive segments, strung together by blobs (Blasdel, 1992). A careful consideration of these patterns, and the information they convey, has led to conclusions (1) that orientation preferences can never be inferred from responses to a single orientation, and (2) that orientation preferences are not even defined unless responses to several orientations can be compared.

The number of orientation-dependent responses required to determine preferences unambiguously depends on the degree of selectivity since narrow ranges of response require closer intervals of sampling. While an optimal number of differential images has yet to be established, the minimum number is at least $t w o$, since the ambiguities in one differential image (for left or right oblique in a differential image of vertical/horizontal, e.g.) can only be resolved by another differential image (for left/right oblique). Since each differential image reflects responses to two (orthogonal) orientations, a minimum of four 2DG autoradiograms would be required to provide the same information.

\section{Linearity of orientation preference}

The most striking aspect of Hubel and Wiesel's (1974a) earlier work was the linearity with which orientation preferences were observed to rotate laterally. Linear zones are apparent with twodimensional mapping as well, where they appear as two-dimensional patches of rainbow (Blasdel and Salama, 1986). As one can see in Figure $7 a$, the iso-orientation slabs within each patch correspond exactly to those expected from Hubel and Wiesel's illustration in Figure $1 c$, but only in areas $0.5-1.0 \mathrm{~mm}$ across, circumscribed by sharply delineated boundaries where linearity breaks down.

\section{Singularities}

Singularities arise whenever orientation preferences rotate continuously through $\pm 180^{\circ}$ along closed paths in two dimensions. They were evident in the first optical recordings from monkey striate cortex (Blasdel and Salama, 1986), and they were shown to correlate with cytochrome oxidase blobs shortly thereafter (Blasdel and Tootell, 1987; Blasdel, 1989a). They are likely to be a general feature of striate cortical organization (Swindale, 1992), since they recently have also been observed in cats (Bonhoeffer and Grinvald, 1991). Because they frequently accompany fractures in monkey striate cortex, it is not clear whether these two organizations should be considered separately or as parts of a continuum. The distinction introduced here, that fractures and singularities reflect gradient discontinuities of less and more than $90^{\circ}$, can be applied operationally since discontinuities smaller than or equal to $90^{\circ}$ must extend along lines (in a continuous, two-dimensional space), while discontinuities greater than $90^{\circ}$ can only exist at points.

Singularities arise from incremental rotations through angles of symmetry, along closed paths. Hence, vectors produce singularities when they rotate through $360^{\circ}$, while triangles produce them when they rotate through $120^{\circ}$ (Penrose, 1979). The fact 
that singularities in orientation preference arise for rotations through $180^{\circ}$ therefore suggest (1) that orientation preferences rotated through $180^{\circ}$ are equivalent; (2) that they have no directional components, like vectors (that have to rotate through $360^{\circ}$ ); and (3) that directional preferences are not organized laterally in relation to orientation preference. All of these suggestions agree with previous observations (Hubel and Wiesel, 1962; Schiller et al., 1976; G. G. Blasdel, unpublished observations) that neurons preferring opposite directions of movement (i.e., directions offset by $180^{\circ}$ ) are frequently recorded at the same time.

Another consequence of $180^{\circ}$ rotations is that positive "loop"type, as well as negative "tri-radius"-type singularities ensure (1) that preferences for all orientations are brought uniquely close together, within easy reach of most upper-layer dendrites, and (2) that orientation preferences on opposite sides are always perpendicular.

\section{Linearity and selectivity}

Since the apparent selectivity for orientation dips near singularities and fractures, it is tempting to conclude that it correlates with linearity. If orientation selectivity were enhanced through lateral inhibition (Blakemore and Tobin, 1972; Sillito, 1979), for example, it should be more pronounced in regions where the preferences of neighboring cells change most linearly with distance. A correlation is also expected from the close association between gradient discontinuities and blobs, because orientation selectivity is reduced in the latter (Livingstone and Hubel, 1984a). Some caution is advisable, however, since the reduced orientation selectivity that seems apparent could also be induced by the spread of optical signals across fractures.

Such effects can be minimized in future experiments by using larger magnifications to reduce the effects of lateral averaging, and by using confocal optics to reduce scatter. This is probably not the principle explanation for zones of reduced orientation selectivity near fractures, however, since these zones are not distributed evenly around discontinuities, and since they come in a variety of widths and are frequently asymmetric. Preliminary correlations with single-unit recordings also indicate they are populated by less selective cells.

\section{Ocular dominance}

Singularities and fractures are especially abundant in the centers of ocular dominance columns (see Figs. $8 b$, 9; Table 1). The significance of this is difficult to appreciate in the context of only two categories of ocular dominance represented in slabshaped domains. When ocular dominance is represented to reflect binocularity in the upper layers, however, as it is in Figure 17 , it becomes clear that, between the centers of adjacent ocular dominance columns, ocular dominance changes linearly as well. In fact, the perpendicular trajectories of most orientation slabs (see Fig. 12a) ensures that overlapping maps of orientation and ocular dominance change linearly along orthogonal axes. This superposition of orthogonal maps, in the most binocular regions of cortex that express the greatest selectivity for orientation, hardly seems a coincidence and suggests that these linear zones engage in special visual functions.

\section{Linearity and density of orientation preferences}

As illustrated in Figure 10, $c$ and $d$, there is a tradeoff between linearity and density in the mapping of orientation preference. When linearity is optimized, orientation preferences are defined with great resolution, but at low density because large areas of cortex are needed to represent all possibilities. With the optimization of density, on the other hand, all orientation preferences are brought uniquely close together, at the sacrifice of linearity and resolution. As one can see in Figure 10, $c$ and $d$, both schemes are optimized periodically, at complimentary intervals, such that the advantages conferred by each are distributed evenly.

What these advantages are remains to be determined. In view of the observation that upper-layer dendrites are nearly uniform in size, forming cylinders $250-350 \mu \mathrm{m}$ across (Lund and Yoshioka, 1991), an important clue would appear to lie in the range of orientations available to each cell. In the linear zones, each neuron could expect to receive linearly organized, orientationally selective, and binocular information over a small range of orientations. And while this would enable precise judgements about edge position and orientation, within the sampled range, information about orthogonal orientations would be unavailable. The convergence of orientation preferences near singularities, on the other hand, would facilitate comparisons of edge contributions, at different orientations, even though information about single edges would be less precise.

\section{Speculations}

These specializations seem ideal for scene segmentation, where contours are distinguished from surfaces, on the basis of local information. Contours, which are defined here as single, onedimensional edges with well-defined positions and axes, must be distinguished from surfaces, defined here as two-dimensional distributions of edges (or colors), because they are more likely to represent boundary contours (Cohen and Grossberg, 1984; Grossberg, 1987). Since contours and surfaces may both contain edges, however, the distinction is not trivial. If striate cells could exploit the information conveyed locally by dimensionality (e.g., whether features distribute in one or two dimensions) to achieve a partial segmentation, however, the tasks of subsequent areas would be simpler.

Provided this occurs, contours are most likely to be perceived in the linear zones, where orientation preferences are mapped linearly with distance (Fig. 10d). The significance of linear, twodimensional maps of orientation is illustrated by the Hough transform in Figure 18 (Hough, 1962; Barlow, 1980; Ballard and Brown, 1982; Ballard et al., 1983), which was invented to find lines of bubbles in particle detectors, and which works by extracting straight lines from noise (see Fig. 18). It begins by grouping neighboring "bubbles" (Fig. 18a) into pairs and calculating new coordinates, specified by the orientation of an infinitely long line that passes through both points, and the distance between that line and some origin (which, in Fig. 18a, lies at the center). When all point pairs have been transformed into new coordinates, and plotted against orthogonal axes, the detection of straight lines becomes trivial, since all the pairs generated by the same particle track (or contour) lie along the same infinitely long line, and therefore map into the same location (Fig. 18b).

The great benefit of the Hough representation (Fig. 18b), where orientation and position are mapped linearly along orthogonal axes, is that most similar contours become represented by nearest neighbors. Small amounts of noise in the perceived orientation and position of an edge (Fig. 18c) lead to small amounts of scatter about the horizontal and vertical axes (Fig. 18d). But because orientation and position are both mapped linearly, the 


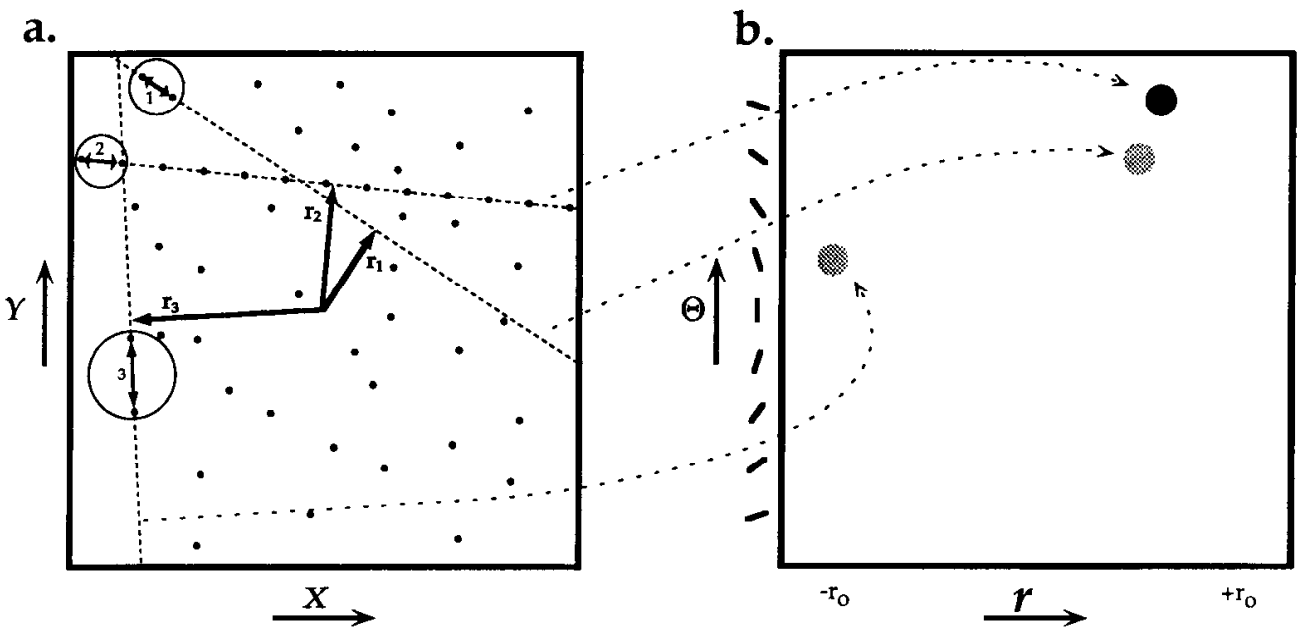

c.

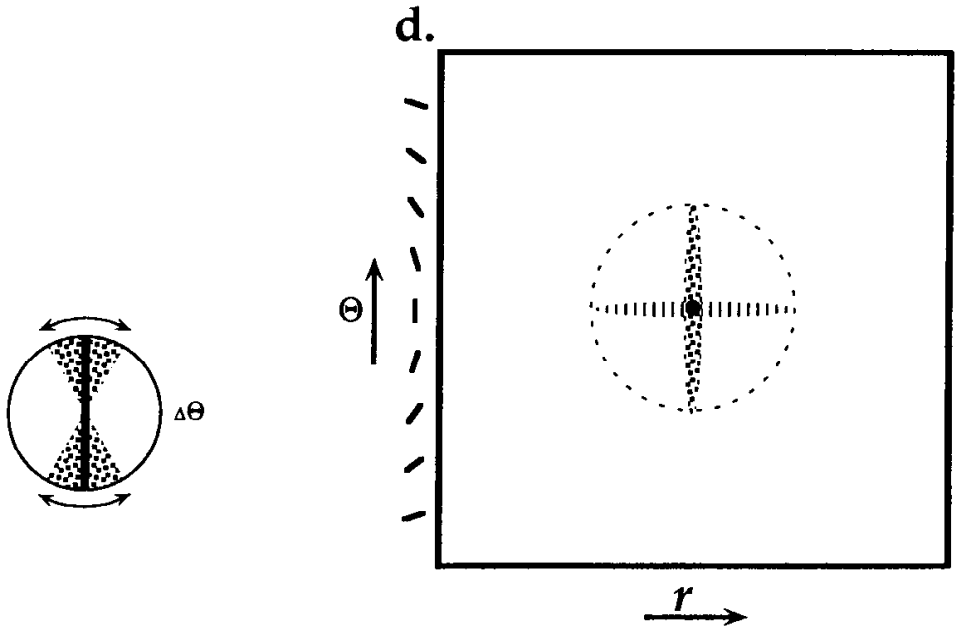

Figure 18. The Hough transform (Hough, 1962) was invented to automate the detection of particle tracks in bubble chambers. Since the task at hand resembles that of contour detection in noisy visual environments (Ballard and Brown, 1982; Ballard et al., 1983), and since orientation is mapped linearly with respect to distance in Hough space as well as in striate cortex, it provides a compelling metaphor for the operations likely to be carried out in striate cortex. $a$, The problem, illustrated here schematically, is to distinguish between bubbles in a straight line, that may indicate a particle track, and those occurring randomly. The transform works by taking the position of each bubble and comparing it with that of its nearest neighbor to find the orientation and displacement of an infinitely long (broken) line passing through both locations. These values specify a unique location in a new "Hough" space (b) where orientation and position are mapped linearly along orthogonal axes. Three sample pairs are indicated by circles in $a$, where the infinitely long lines running through them are indicated by broken lines corresponding to distinct locations in $b$. $b$, When this operation is performed iteratively, on every point in the image, values build up rapidly at "Hough" coordinates corresponding to particle tracks because the bubbles associated with them lie along the same infinitely long lines. It is obvious in $a$, for example, that bubbles corresponding to $r_{2}$ are much more numerous than those corresponding to $r_{1}$ or $r_{3}$, and that consequently they give rise to a larger value (indicated by a darker circle) in $b$. c. The power of this transform derives from the linearity of maps for orientation and position. Because of this, positional uncertainty (indicated by vertical solid bars, upper left aperture) leads to scatter about the horizontal axis (see $d$ ), while orientational uncertainty (indicated by stippling, lower right aperture) registers as scatter about vertical. $d$, Since orientation and position axes are orthogonal, however, values arising from a degraded contour cluster around a central point representing the best possible fit for the observed contour. What has happened, therefore, is that single contours are represented as single points rather than one-dimensional strings of points.

cluster spreads symmetrically about the same point, which corresponds to a single "best" contour.

As Ballard et al. (1983) pointed out, the Hough transform provides a useful metaphor for striate cortex since both engage in similar tasks - contour detection - and both are characterized by linear maps of orientation preference along one axis. The relevance of Hough transforms is strengthened by the recent finding (Blasdel and Salama, 1986) that linear organizations of orientation preference are confined to patches $0.5-1.0 \mathrm{~mm}$ across. But the likelihood that cells in the linear zones use Hough transforms to find edges rests on the supposition that position is mapped linearly along the orthogonal axis.
This still must be demonstrated. But the orthogonality between orientation and ocular dominance makes it likely. This is because the duplicate representations of retinotopic space in the ocular dominance bands of layer 4c (Hubel et al., 1974; Blasdel and Fitzpatrick, 1984) require that receptive field positions move twice as quickly across them as along them, thereby creating small linear maps in the appropriate directions. And since these representations are relayed vertically upward, with little or no distortion (Fitzpatrick et al., 1985), they are at least available in the upper layers.

The orientation preferences near singularities offer different constraints (Fig. 10c). All orientations are represented close to 
a

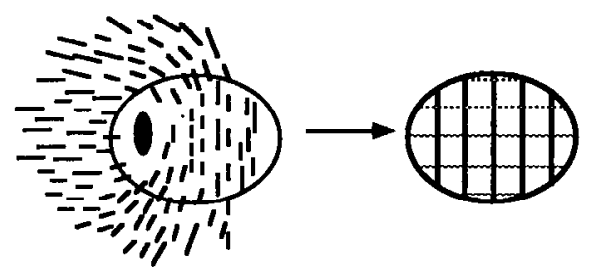

b

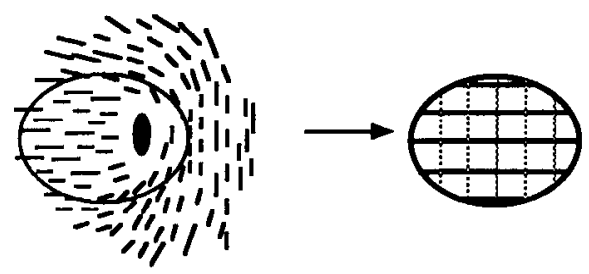

C

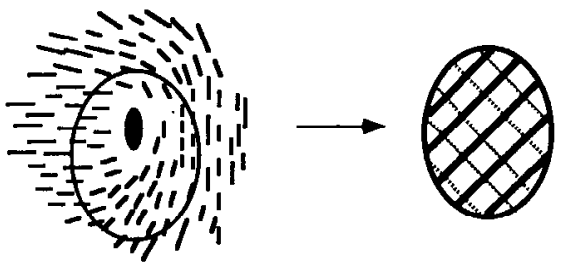

Figure 19. This figure illustrates one possible implementation of texture space near singularities. Because the dendritic fields of most upper layer neurons are contained within cylindrical zones, 250-350 $\mu \mathrm{m}$ across (or narrower), the spectrum of orientations impinging on each cell may well depend on those available within each zone. If this is the case, the unique arrangements of orientation preferences around singularities may generate continuous maps of edge density (with special emphasis on edges that are perpendicular) in populations of postsynaptic cells. Consider the tight loop of orientation preferences around the positive singularity in this figure. Dendritic fields enclosing this singularity can be expected to receive some information about all orientations. But those displaced toward the right $(a)$ receive more inputs preferring horizontal than vertical, which could impose a preference for higher densities of horizontal edges in two-dimensional textures (keep in mind that the selectivities for orientation and position are both reduced). Similarly, the dendritic fields illustrated in $b$ and $c$ might be expected to receive more inputs for vertical and left oblique, implying preferences for higher densities of edges at these orientations (as opposed to others). In this simple fashion, a continuous coarse coding of all possible textures might be achieved for one part of visual space, with the advantage that most similar textures are represented by nearest neighbors.

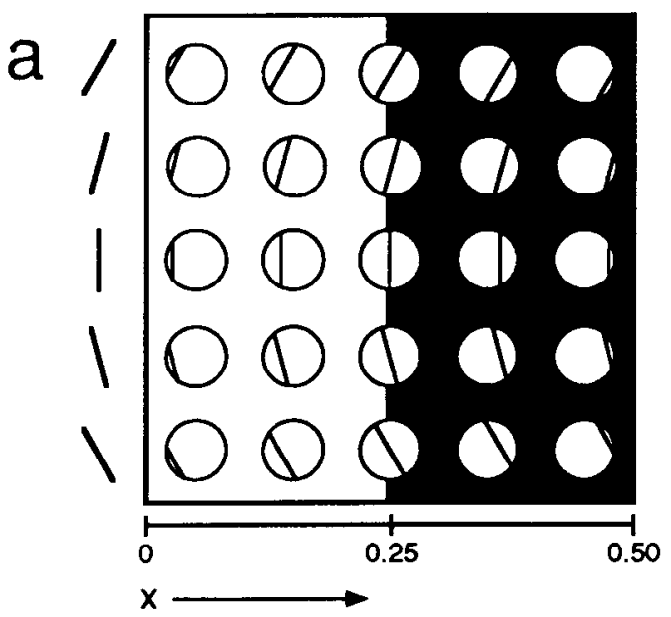

b

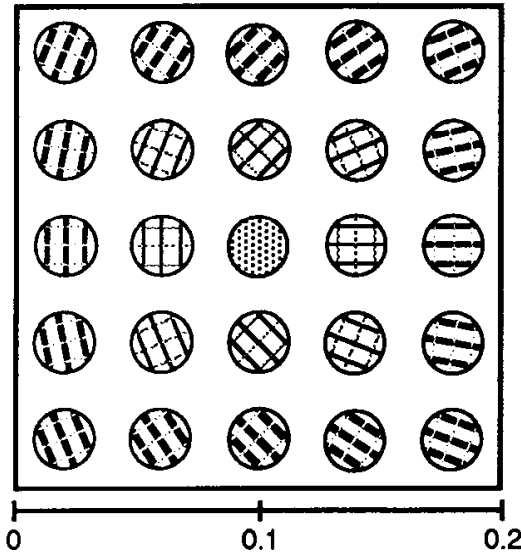

Figure 20. These two drawings illustrate the manner in which laterally segregated and complementary regions of cortex might engage in scene segmentation, by specializing in the detection of contours and surfaces separately. $a$, The continuous patches lie between the centers of ocular dominance bands (see inset) and overlap with regions where binocularity, orientation selectivity, and the continuity of ocular dominance are most pronounced. According to the proposed hypothesis, cells within these zones might specialize in contour detection. Because orientation preferences rotate continuously along one axis (in this case vertical), and lateral displacements (might) change continuously along the other, most similar contours (for one part of visual space) are represented by nearest neighbors-a scheme that (along with Hough transforms) confers decided advantages in the isolation of single-edge contours from noisy visual environments. Note also, that since none of the neurons in these zones need (or receive) information about all orientations in any case, complete cycles through $180^{\circ}$ are unnecessary; the important advantages arise from linearity and continuity. $b$, From the example in Figure 19, it is apparent that singularities may provide arrangements of orientation preference needed for orderly representations of edge texture, where different combinations of edges at orthogonal orientations might be mapped linearly. A more exhaustive illustration of this organization is provided in $b$.. 
one another, so every cell is likely to receive inputs preferring all possible orientations. And the arrangement of orientation preferences around them, such that perpendicular orientations are always represented on opposite sides, should be ideal for comparing edge contributions (or densities) at orthogonal orientations, which is necessary in the evaluation of textures. If one considers the orientations represented within a $150-\mu \mathrm{m}$ wide disk of dendrites that is displaced gradually across a positive "loop"-type singularity, it is easy to see how ratios of orthogonally oriented edges could vary linearly with position. In Figure 19, $a$ and $b$, for example, one sees that the ratio of vertical to horizontal edges varies linearly with displacements along the $x$-axis, while the ratio of left to right oblique edges varies linearly with displacements along the y-axis (Fig. 19c). While important details clearly remain to be worked out, this scheme would allow most similar textures to be encoded by nearest neighbors, in a two-dimensional feature space, with ratios of vertical/horizontal and left/right oblique edges represented along orthogonal axes, such that Hough-like principles might be exploited in the analysis of textures (Fig. 20b).

\section{Hypothesis}

These observations may be taken to suggest a general hypothesis of striate function: (1) that laterally segregated regions of cortex specialize in the detection of contours and surfaces, (2) that linear zones engage primarily in contour detection, and (3) that regions around singularities specialize in surface textures. This hypothesis is consistent with many existing observations. It can explain, for example, the long-term elevations in metabolic activity indicated by increased levels of cytochrome oxidase (WongRiley, 1979) near singularities and regions specializing in color (Livingstone and Hubel, 1984a; Ts'o and Gilbert, 1988), if one considers that surfaces occupy most of visual space and that the cortical regions responsible for analyzing them are disproportionately small.

A key feature of this hypothesis is that laterally segregated cortical circuits exploit dimensionality in distinguishing between contours and surfaces. It therefore can be tested by seeing whether or not this is true. In order for it to be contour detecting cells should be concentrated in the linear zones, and they should be present in greater numbers since these zones occupy most of the cortical surface. They should be mapped linearly for position as well as for orientation (see Fig. 20a). And, they might summate linearly (to longer edges) but not spatially (to multiple edges distributed in two dimensions) in their receptive fields. Interactions like this have already been observed (Van Essen et al., 1989; Born and Tootell, 1991; von der Heydt et al., 1992), but they need to be explored in greater detail.

Cells specializing in surfaces, on the other hand, should bc located near singularities. They should be less selective for position and orientation, and they should summate in two dimensions, which means they should respond better to gratings than to single edges. In fact, they are likely to be characterized by selectivity for something resembling spatial frequency. If the scheme illustrated in Figure 19 has merit, they should be especially sensitive to the spatial frequencies of crossed gratings presented simultaneously (see Fig. 20b).

If striate cortex can exploit dimensionality to distinguish locally between contours and surfaces, it solves a major dilemma for cells in subsequent areas, which are thereby freed to pursue more sophisticated perceptions. If so, this could explain why, in addition to being the first visual area in cortex, striate cortex is also the largest visual area, comprising some $10-15 \%$ of the entire neocortex in macaque monkeys (Van Essen, 1984), and the only one to recompute all visual information before subsequent processing.

\section{References}

Albrecht DG, De Valois RL, Thorell LG (1980) Visual cortical neurons: are bars or gratings the optimal stimuli? Science 207:88-90.

Ballard D, Hinton J, Sejnowski T (1983) Parallel visual computation. Nature 306:5938:21-26.

Ballard DH, Brown CM (1982) Computer vision. Englewood Cliffs, NJ: Prentice-Hall.

Barlow HB (1980) Critical limiting factors in the design of the eye and visual cortex. Proc R Soc Lond [Biol] 212:1-34.

Blakemore C, Tobin EA (1972) Lateral inhibition between orientation detectors in the cat's visual cortex. Exp Brain Res 15:439-440.

Blasdel GG (1989a) Topography of visual function as shown with voltage sensitive dycs. In: Sensory systems in the mammalian brain (Lund JS, ed), pp 242-268. New York: Oxford UP.

Blasdel GG (1989b) Visualization of neuronal activity in monkey striate cortex. Annu Rev Physiol 51:561-581.

Blasdell GG (1992) Differential imaging of ocular dominance and orientation selectivity in monkey cortex. J Neurosci 12:3117-3140.

Blasdel GG, Fitzpatrick (1984) Physiological organization of layer 4 in macaque striate cortex. J Neurosci 4:880-895.

Blasdel GG, Haglund MM (1989) Bicuculine induced disruption of optically determined ocular dominance bands in macaque striate cortex. Soc Neurosci Abstr 15:320.10.

Blasdel GG, Lund JS (1982) Termination of afferent axons in macaque striate cortex. J Neurosci 3:1389-1413.

Blasdel GG, Salama G (1986) Voltage-sensitive dyes reveals a modular organization in the monkey striate cortex. Nature 321:579-585.

Blasdel GG, Tootell RBH (1987) Studies of orientation and motion selectivity in monkey striate cortex using optical techniques. Soc Neurosci Abstr 13:2.

Bonhoeffer T, Grinvald A (1991) Iso-orientation domains in cat visual cortex are arranged in pinwheel-like patterns. Nature 353:429-431.

Born RT, Tootell RBH (1991) Single unit and 2-deoxyglucose studies of side inhibition in macaque striate cortex. Proc Natl Acad Sci USA, in press.

Cohen MA, Grossberg S (1984) Neural dynamics of brightness perception: features, boundaries, diffusion, and resonnance. Percept Psychophys 36:428-456.

DeValois RL, Albrecht DG, Thorell LG (1982) Spatial frequency selectivity of cells in macaque visual cortex. Vision Res 22:545-559.

Fitzpatrick D, Lund JS, Blasdel GG (1985) Intrinsic connections of macaque striate cortex: afferent and efferent connections of lamina 4C. J Neurosci 5:3329-3349.

Grinvald A, Lieke E, Frostig RP, Gilbert C, Wiesel RM (1986) Functional architecture of cortex revealed by optical imaging of intrinsic signals. Nature $324: 351-364$

Grossberg S (1987) Cortical dynamics of three-dimensional form, color, and brightness perception: I. Monocular theory. Percept Psychophys $41: 87-116$.

Haglund MM, Blasdel GG (1991) Role of activity in generation of upper layer ocular dominance columns in monkey striate cortex. Soc Neurosci Abstr 17:431.2.

Hendrickson AE, Wilson JR, Ogren MP (1978) The neuroanatomical organization of pathways between the dorsal lateral geniculate nucleus and visual cortex in old world and new world primates. J Comp Neurol 182:123-136.

Horton JC, Hubel DH (1980) Regular patchy distribution of cytochrome oxidase staining in primary visual cortex of macaque monkey. Nature 292:762-764.

Hubel DH, Wiesel TN (1962) Receptive fields, binocular interaction and functional architecture of monkey striate cortex. J Physiol (Lond) 160:106-154.

Hubel DH, Wiesel TN (1968) Receptive fields and functional architecture of monkey striate cortex. J Physiol (Lond) 195:21 5-243.

Hubel DH, Wiesel TN (1972) Laminar and columnar distribution of geniculo-cortical fibers in the macaque monkey. J Comp Neurol 146: 421-450.

Hubel DH, Wiesel TN (1974a) Sequence regularity and geometry of 
orientation columns in the monkey striate cortex. J Comp Neurol 158:267-293.

Hubel DH, Wiesel TN (1974b) Uniformity of monkey striate cortex: a parallel relationship between field size, scatter and magnification factor. J Comp Neurol 158:295-306.

Hubel DH, Wiesel TN (1977) Functional architecture of macaque monkey visual cortex. Proc Roy Soc Lond B 198:1-59.

Hubel DH, Weisel TN, LeVay S (1974) Visual field of representation in layer IVC of monkey striate cortex. Soc Neurosci Abstr X:264.

Hubel DH, Wiesel TN, Stryker MP (1978) Anatomical demonstration of orientation columns in macaque monkey. J Comp Neurol 177: 361-380.

Humphrey AL, Hendrickson AE (1983) Background and stimulusinduced patterns of high metabolic activity in the visual cortex area (area 17) of the squirrel and macaque monkey. J Neurosci 3:345-358.

Kiorpes L, Blasdel GG (1987) Optical studies of macaque striate cortex during development. Soc Neurosci Abstr 13:1243.

Kulikowski JJ, Bishop PO (1981) Fourier analysis and spatial representation in the visual cortex. Experientia 37:160-163.

livingstone MS, Hubel DH (1984a) Anatomy and physiology of a color system in the primate visual cortex. J Neurosci 4:309-356.

Livingstone MS, Hubel DH (1984b) Specificity of intrinsic connections in primate primary visual cortex. J Neurosci 4:2830-2835.

Lund JS (1973) Organization of neurons in the visual cortex, area 17, of the monkey (Macaca mulatta). J Comp Neurol 147:445-496.

Lund JS, Boothe RG (1975) Interlaminar connections and pyramidal neuron organization in the visual cortex, area 17, of the macaque monkey. J Comp Neurol 159:305-334.

Lund JS, Yoshioka T (1991) Local circuit neurons of macaque monkey striate cortex. III. Neurons of laminae 4B, 4A and 3B. J Comp Neurol 311:234-258.

Movshon JA, Thompson ID, Tolhurst DJ (1978a) Spatial summation in the receptive fields of simple cells in the cat's striate cortex. J Physiol (Lond) 283:53-77.

Movshon JA, Thompson ID, Tolhurst DJ (1978b) Receptive field organization of complex cells in the cat's striate cortex. J Physiol 283: $79-99$.

Obermayer K, Schulten K, Blasdel GG (1992) A comparison between a neural network model for the formation of brain maps and experimental data. Theor Biophys Tech Rep UIUC-B1-TB-92-01.

Orbach HS, Van Essen D (1986) Optical mapping of activity in primate visual cortex. Nature 321:564-565.

Penrose R (1979) The topology of ridge systems. Ann Hum Genet 42: 435-444.

Pollen DA, Ronner SF (1982) Spatial computation performed by sim- ple and complex cells in the visual cortex of the cat. Vision Res 22: 101-118.

Rockland KS, Lund JS (1983) Intrinsic laminar lattice connections in primate visual cortex. J Comp Neurol 216:303-318.

Schiller PH, Malpeli JG (1978) Functional specificity of lateral geniculate nucleus laminae of the rhesus monkey. J Neurophysiol 41:788797.

Schiller PH, Finlay BL, Volman SF (1976) Quantitative studies of single cell properties in monkey striate cortex. I. Spatiotemporal organization of receptive fields. J Neurophysiol 39:1288-1319.

Schiller PH, Finlay BL, Volman (1976) Quantitative studies of singlecell properties in monkey striate cortex. III. Spatial frequency. J Neurophysiol 39:1334-1351.

Schoppmann A, Stryker MP (1981) Physiological evidence that the 2-deoxyglucose method reveals orientation in cat visual cortex. $\mathrm{Na}$ ture 293:574-576.

Sillito AM (1979) Inhibitory mechanisms influencing complex cell orientation selectivity and their modification at high resting discharge levels. J Physiol (Lond) 229:33-53.

Sokoloff L, Reivich M, Kennedy C, Des Rosiers MH, Patlak CS, Pettigrew KD, Sakurada O, Shinohara M (1977) The $\left[{ }^{14} \mathrm{C}\right]$ deoxyglucose method for measurement of local cerebral glucose utilization theory, procedure, and normal values in the conscious and anesthesized rat. J Neurochem 28:897-916.

Swindale N (1992) A model for the coordinated development of columnar systems in primate striate cortex. Biol Cyber 66:217-230.

Ts'o DY, Gilbert CD (1988) The organization of chromatic and spatial interactions in the primate striate cortex. J Neurosci 8:1712-1727.

Ts'o DY, Frostig RD, Lieke EE (1990) Functional organization of primate visual cortex revealed by high resolution optical imaging. Science 249:417-420.

Van Essen DC (1984) Functional organization of primate visual cortex. In: Cerebral cortex, Vol 3, Visual cortex (Peters A, Jones EG, ed), pp 259-329. New York: Plenum.

Van Essen DC, DeYoe EA, Olavarria JF, Knierim JJ, Fox JM, Sagi D, Julesz B (1989) Neural responses to static and moving texture patterns in visual cortex of the macaque monkey. In: Neural mechanisms of visual perception (Lam DMK, Gilbert C, eds), pp 137-154. Woodlands, TX: Portfolio.

von der Heydt R, Peterhans E, Dursteler MR (1992) Periodic patternselective cells in monkey visual cortex. J Neurosci 12:1416-1434.

Wong-Riley MTT (1979) Changes in the visual system of monocularly sutured or enucleated cats demonstrable with cytochrome oxidase histochemistry. Brain Res 171:11-28. 